\title{
Dissection of the mechanism of the Wittig reaction
}

\author{
Paola Farfán, ${ }^{\dagger}$ Sara Gómez, ${ }^{\ddagger}$ Albeiro Restrepo ${ }^{* \dagger}$ \\ ${ }^{\dagger}$ Instituto de Química, Universidad de Antioquia UdeA, Calle 70 No. 52-21, Medellín, \\ Colombia \\ ${ }^{\ddagger}$ Scuola Normale Superiore, Classe di Scienze, Piazza dei Cavalieri 7, 56126, Pisa, Italy \\ E-mail: albeiro.restrepo@udea.edu.co \\ Supporting Information
}

\section{Contents}

$1 \quad$ Non-Stabilized Ylides $\left(\mathrm{Ph}_{3} \mathrm{PCHMe}+\mathrm{MeCHO}\right) \quad$ S2

2 Stabilized Ylides $\left(\mathrm{Me}_{3} \mathrm{PCHCOOMe}+\mathrm{PhCHO}\right) \quad$ S20

3 Semi-Stabilized Ylides ( $\left.\mathrm{Me}_{3} \mathrm{PCHPh}+\mathrm{PhCHO}\right) \quad$ S35 


\section{Non-Stabilized Ylides $\left(\mathrm{Ph}_{3} \mathrm{PCHMe}+\mathrm{MeCHO}\right)$}

\begin{tabular}{|c|c|c|c|}
\hline \multicolumn{4}{|c|}{$\begin{array}{l}\mathrm{Ph}_{3} \mathrm{PCHMe} \\
\mathrm{E}(\mathrm{B} 3 \mathrm{LYP} 6-31 \mathrm{G}(\mathrm{d})) \text { Energy }=-1114.85 \\
\text { Number of imaginary frequencies }=0\end{array}$} \\
\hline $\mathrm{C}$ & 0.292372 & 0.233511 & 2.436467 \\
\hline $\mathrm{P}$ & 0.055536 & 0.051136 & 0.780325 \\
\hline $\mathrm{H}$ & -0.477616 & 0.827850 & 2.923896 \\
\hline $\mathrm{C}$ & -2.546797 & -3.472876 & -0.885814 \\
\hline $\mathrm{C}$ & -2.368096 & -2.336629 & -1.678668 \\
\hline $\mathrm{C}$ & -1.588552 & -1.275345 & -1.214901 \\
\hline $\mathrm{C}$ & -0.978002 & -1.335755 & 0.048624 \\
\hline $\mathrm{C}$ & -1.177194 & -2.475561 & 0.839311 \\
\hline $\mathrm{C}$ & -1.950214 & -3.541187 & 0.374429 \\
\hline $\mathrm{H}$ & -3.155500 & -4.297961 & -1.246590 \\
\hline $\mathrm{H}$ & -2.839310 & -2.275391 & -2.656607 \\
\hline $\mathrm{H}$ & -1.462990 & -0.392799 & -1.837203 \\
\hline $\mathrm{H}$ & -0.733844 & -2.517278 & 1.831102 \\
\hline $\mathrm{H}$ & -2.093309 & -4.419899 & 0.998192 \\
\hline $\mathrm{C}$ & 4.179815 & -0.293607 & -1.345872 \\
\hline $\mathrm{C}$ & 3.096878 & -1.010907 & -1.856803 \\
\hline $\mathrm{C}$ & 1.849433 & -0.938392 & -1.234718 \\
\hline $\mathrm{C}$ & 1.669915 & -0.141308 & -0.094491 \\
\hline $\mathrm{C}$ & 2.766831 & 0.573326 & 0.414788 \\
\hline $\mathrm{C}$ & 4.013260 & 0.497026 & -0.207075 \\
\hline $\mathrm{H}$ & 5.150771 & -0.354098 & -1.830765 \\
\hline $\mathrm{H}$ & 3.223017 & -1.634119 & -2.738278 \\
\hline $\mathrm{H}$ & 1.020226 & -1.512215 & -1.634335 \\
\hline $\mathrm{H}$ & 2.625485 & 1.176109 & 1.307406 \\
\hline $\mathrm{H}$ & 4.854470 & 1.052105 & 0.199859 \\
\hline $\mathrm{C}$ & -2.136828 & 3.879597 & -0.649781 \\
\hline $\mathrm{C}$ & -2.843431 & 2.855833 & -0.016318 \\
\hline $\mathrm{C}$ & -2.185607 & 1.688560 & 0.373809 \\
\hline $\mathrm{C}$ & -0.807344 & 1.542222 & 0.151443 \\
\hline $\mathrm{C}$ & -0.105459 & 2.575399 & -0.484386 \\
\hline $\mathrm{C}$ & -0.768530 & 3.735467 & -0.886583 \\
\hline $\mathrm{H}$ & -2.651233 & 4.785212 & -0.960357 \\
\hline $\mathrm{H}$ & -3.909139 & 2.961473 & 0.168799 \\
\hline $\mathrm{H}$ & -2.746793 & 0.888452 & 0.847886 \\
\hline $\mathrm{H}$ & 0.960562 & 2.475249 & -0.663931 \\
\hline $\mathrm{H}$ & -0.214477 & 4.527495 & -1.383391 \\
\hline
\end{tabular}




$\begin{array}{llll}\mathrm{C} & 1.154872 & -0.656460 & 3.304850 \\ \mathrm{H} & 1.944265 & -1.150815 & 2.724174 \\ \mathrm{H} & 1.662988 & -0.068704 & 4.083578 \\ \mathrm{H} & 0.605445 & -1.455204 & 3.831820\end{array}$

\section{$\mathrm{MeCHO}$}

E(B3LYP 6-31G(d)) Energy=-153.830121079 Hartrees

Number of imaginary frequencies $=0$

$\begin{array}{cccc}\mathrm{C} & 0.233858 & 0.399833 & -0.000036 \\ \mathrm{H} & 0.302305 & 1.511872 & 0.000048 \\ \mathrm{O} & 1.237382 & -0.277117 & 0.000008 \\ \mathrm{C} & -1.170647 & -0.148549 & -0.000013 \\ \mathrm{H} & -1.154378 & -1.240788 & -0.000735 \\ \mathrm{H} & -1.712785 & 0.218453 & 0.881435 \\ \mathrm{H} & -1.713467 & 0.219696 & -0.880513\end{array}$

Cis Adduct $\left(\mathrm{Ph}_{3} \mathrm{PCHMe}+\mathrm{MeCHO}\right)$

E(B3LYP 6-31G(d)) Energy=-1268.69476831 Hartrees

Number of imaginary frequencies $=0$

$\begin{array}{lccc}\mathrm{O} & 4.47075 & 1.06252 & -1.11186 \\ \mathrm{C} & 4.20493 & 0.16329 & -1.88246 \\ \mathrm{P} & -0.24443 & -0.00380 & -0.35262 \\ \mathrm{C} & 0.81990 & -0.21498 & -1.66749 \\ \mathrm{C} & -2.06169 & 0.24443 & -0.68893 \\ \mathrm{C} & -2.87008 & -0.86151 & -1.00720 \\ \mathrm{C} & -4.16589 & -0.68631 & -1.49445 \\ \mathrm{C} & -4.67760 & 0.59838 & -1.68245 \\ \mathrm{C} & -3.87967 & 1.70664 & -1.38980 \\ \mathrm{C} & -2.58297 & 1.53182 & -0.90750 \\ \mathrm{C} & 0.26746 & 1.44680 & 0.64093 \\ \mathrm{C} & -0.20709 & -1.47097 & 0.76433 \\ \mathrm{C} & 1.54091 & 2.00480 & 0.45768 \\ \mathrm{C} & 1.95727 & 3.07654 & 1.24865 \\ \mathrm{C} & 1.11983 & 3.58810 & 2.24077 \\ \mathrm{C} & -0.14158 & 3.02372 & 2.44262 \\ \mathrm{C} & -0.56590 & 1.95882 & 1.64864 \\ \mathrm{C} & 0.94520 & -2.26922 & 0.76662 \\ \mathrm{C} & 1.05863 & -3.34600 & 1.64626\end{array}$




$\begin{array}{lccc}\mathrm{C} & 0.02952 & -3.62584 & 2.54700 \\ \mathrm{C} & -1.11458 & -2.82554 & 2.56407 \\ \mathrm{C} & -1.23397 & -1.75494 & 1.67737 \\ \mathrm{H} & 3.58674 & 0.35421 & -2.78467 \\ \mathrm{H} & 1.08530 & 0.74299 & -2.11736 \\ \mathrm{H} & -2.48902 & -1.86945 & -0.86745 \\ \mathrm{H} & -4.77559 & -1.55589 & -1.72647 \\ \mathrm{H} & -5.68743 & 0.73555 & -2.05980 \\ \mathrm{H} & -4.26712 & 2.71147 & -1.53785 \\ \mathrm{H} & -1.97292 & 2.40539 & -0.69705 \\ \mathrm{H} & 2.21395 & 1.59520 & -0.28641 \\ \mathrm{H} & 2.94518 & 3.49966 & 1.09000 \\ \mathrm{H} & 1.45010 & 4.41776 & 2.86043 \\ \mathrm{H} & -0.79665 & 3.41134 & 3.21835 \\ \mathrm{H} & -1.55175 & 1.53304 & 1.81096 \\ \mathrm{H} & 1.74701 & -2.02257 & 0.07691 \\ \mathrm{H} & 1.95393 & -3.96205 & 1.63277 \\ \mathrm{H} & 0.12004 & -4.46075 & 3.23695 \\ \mathrm{H} & -1.91679 & -3.03525 & 3.26667 \\ \mathrm{H} & -2.13342 & -1.14705 & 1.69253 \\ \mathrm{C} & 4.68192 & -1.25844 & -1.73032 \\ \mathrm{H} & 3.81515 & -1.93017 & -1.71002 \\ \mathrm{H} & 5.28860 & -1.54314 & -2.60051 \\ \mathrm{H} & 5.26883 & -1.36961 & -0.81552 \\ \mathrm{C} & 0.59976 & -1.35275 & -2.65079 \\ \mathrm{H} & -0.24601 & -1.21000 & -3.34551 \\ \mathrm{H} & 1.49231 & -1.49660 & -3.27600 \\ \mathrm{H} & 0.42419 & -2.30810 & -2.13817\end{array}$

Cis TS1

E(B3LYP 6-31G(d)) Energy= -1268.68304449 Hartrees Imaginary Freq $=-174.7206 \mathrm{~cm}^{-1}$

$\begin{array}{lccc}\mathrm{O} & -3.121005 & 0.219637 & 1.646081 \\ \mathrm{C} & -2.429587 & -0.536453 & 2.383967 \\ \mathrm{P} & 0.171354 & -0.012458 & 0.314320 \\ \mathrm{C} & -0.387162 & -0.393757 & 1.962286 \\ \mathrm{C} & 1.998460 & -0.199874 & 0.170136 \\ \mathrm{C} & 2.565827 & -1.483485 & 0.066384 \\ \mathrm{C} & 3.947651 & -1.660436 & 0.140941 \\ \mathrm{C} & 4.786898 & -0.563231 & 0.337804\end{array}$




\begin{tabular}{|c|c|c|c|}
\hline $\mathrm{C}$ & 4.234197 & 0.711308 & 0.474916 \\
\hline $\mathrm{C}$ & 2.853415 & 0.892054 & 0.401510 \\
\hline $\mathrm{C}$ & -0.248382 & 1.711097 & -0.118715 \\
\hline $\mathrm{C}$ & -0.594261 & -1.037118 & -0.997761 \\
\hline $\mathrm{C}$ & -1.331691 & 2.342271 & 0.512721 \\
\hline $\mathrm{C}$ & -1.684240 & 3.640535 & 0.134246 \\
\hline $\mathrm{C}$ & -0.977608 & 4.308635 & -0.864748 \\
\hline $\mathrm{C}$ & 0.080281 & 3.668125 & -1.515498 \\
\hline $\mathrm{C}$ & 0.439111 & 2.372112 & -1.152306 \\
\hline $\mathrm{C}$ & -1.994910 & -1.166230 & -0.967619 \\
\hline $\mathrm{C}$ & -2.645036 & -1.853973 & -1.992957 \\
\hline $\mathrm{C}$ & -1.919496 & -2.404590 & -3.051284 \\
\hline $\mathrm{C}$ & -0.533303 & -2.252219 & -3.096520 \\
\hline $\mathrm{C}$ & 0.128919 & -1.563710 & -2.079094 \\
\hline $\mathrm{H}$ & -2.173961 & -0.190413 & 3.411729 \\
\hline $\mathrm{H}$ & -0.220724 & 0.538171 & 2.514811 \\
\hline $\mathrm{H}$ & 1.929453 & -2.351803 & -0.074729 \\
\hline $\mathrm{H}$ & 4.365812 & -2.659092 & 0.048836 \\
\hline $\mathrm{H}$ & 5.863031 & -0.701470 & 0.394794 \\
\hline $\mathrm{H}$ & 4.878198 & 1.570502 & 0.641681 \\
\hline $\mathrm{H}$ & 2.442093 & 1.888644 & 0.522812 \\
\hline $\mathrm{H}$ & -1.944323 & 1.800430 & 1.234866 \\
\hline $\mathrm{H}$ & -2.526693 & 4.122731 & 0.622477 \\
\hline $\mathrm{H}$ & -1.256967 & 5.319924 & -1.148817 \\
\hline $\mathrm{H}$ & 0.622689 & 4.174409 & -2.309399 \\
\hline $\mathrm{H}$ & 1.254828 & 1.880319 & -1.673885 \\
\hline $\mathrm{H}$ & -2.573596 & -0.710889 & -0.161062 \\
\hline $\mathrm{H}$ & -3.726743 & -1.952632 & -1.963358 \\
\hline $\mathrm{H}$ & -2.433497 & -2.941080 & -3.844633 \\
\hline $\mathrm{H}$ & 0.036533 & -2.663984 & -3.925168 \\
\hline $\mathrm{H}$ & 1.204457 & -1.437526 & -2.138165 \\
\hline $\mathrm{C}$ & -2.714803 & -2.041467 & 2.361517 \\
\hline $\mathrm{H}$ & -2.042325 & -2.620460 & 3.000575 \\
\hline $\mathrm{H}$ & -3.737123 & -2.176611 & 2.739798 \\
\hline $\mathrm{H}$ & -2.686563 & -2.446986 & 1.346285 \\
\hline $\mathrm{C}$ & 0.349611 & -1.556678 & 2.626522 \\
\hline $\mathrm{H}$ & 1.434447 & -1.409683 & 2.732958 \\
\hline $\mathrm{H}$ & -0.052751 & -1.699562 & 3.636769 \\
\hline $\mathrm{H}$ & 0.198104 & -2.500662 & 2.088675 \\
\hline
\end{tabular}

Cis OP1 
E(B3LYP 6-31G(d)) Energy=-1268.72504868 Hartrees Number of imaginary frequencies $=0$

$\begin{array}{lccc}\mathrm{O} & -0.22136 & -1.91668 & 1.18441 \\ \mathrm{C} & -0.34290 & -1.47594 & 2.53249 \\ \mathrm{P} & -0.05530 & -0.25876 & 0.43484 \\ \mathrm{C} & 0.10072 & -0.01526 & 2.32922 \\ \mathrm{C} & 0.39005 & 1.59807 & 0.09412 \\ \mathrm{C} & 1.63135 & 2.01514 & -0.41049 \\ \mathrm{C} & 1.99434 & 3.36641 & -0.44126 \\ \mathrm{C} & 1.12229 & 4.34075 & 0.03914 \\ \mathrm{C} & -0.11331 & 3.95063 & 0.56157 \\ \mathrm{C} & -0.46354 & 2.60120 & 0.59524 \\ \mathrm{C} & -1.77264 & -0.26328 & -0.28796 \\ \mathrm{C} & 1.12131 & -0.98047 & -0.81537 \\ \mathrm{C} & -2.65495 & -1.31017 & 0.02893 \\ \mathrm{C} & -3.93025 & -1.36658 & -0.53445 \\ \mathrm{C} & -4.34372 & -0.39225 & -1.44272 \\ \mathrm{C} & -3.47014 & 0.63999 & -1.78559 \\ \mathrm{C} & -2.20041 & 0.70690 & -1.21095 \\ \mathrm{C} & 2.06319 & -1.95361 & -0.45621 \\ \mathrm{C} & 2.93241 & -2.48751 & -1.40994 \\ \mathrm{C} & 2.85117 & -2.08018 & -2.74140 \\ \mathrm{C} & 1.90051 & -1.12792 & -3.11467 \\ \mathrm{C} & -1.05226 & -0.57337 & -2.15664 \\ \mathrm{H} & -1.40090 & -1.49633 & 2.84969 \\ \mathrm{H} & -0.63386 & 0.72306 & 2.67274 \\ \mathrm{H} & 2.33745 & 1.28247 & -0.78747 \\ \mathrm{H} & 2.96380 & 3.65203 & -0.84268 \\ \mathrm{H} & -0.80474 & 4.69767 & 0.01312 \\ \mathrm{H} & -1.43330 & 2.32812 & 0.94430 \\ \mathrm{H} & -2.31419 & -2.09216 & 0.69550 \\ \mathrm{H} & -4.59686 & -2.18286 & -0.26846 \\ \mathrm{H} & -5.33535 & -0.43997 & -1.88542 \\ \mathrm{H} & -3.77441 & 1.39949 & -2.50109 \\ \mathrm{H} & -1.5454 & 1.52516 & -1.48515 \\ \mathrm{H} & -2.31337 & 0.56481 \\ \mathrm{H} & -3.23466 & -1.11132 \\ \mathrm{H} & -2.50618 & -3.48586 \\ \mathrm{H} & & & -4.15177 \\ \mathrm{H} & -2.46120\end{array}$




$\begin{array}{llrl}\mathrm{C} & 0.45403 & -2.35891 & 3.48647 \\ \mathrm{H} & 0.34812 & -2.01909 & 4.52416 \\ \mathrm{H} & 0.08414 & -3.38786 & 3.42611 \\ \mathrm{H} & 1.51897 & -2.36967 & 3.23263 \\ \mathrm{C} & 1.48595 & 0.39587 & 2.83161 \\ \mathrm{H} & 1.72067 & 1.41892 & 2.52550 \\ \mathrm{H} & 1.51749 & 0.35730 & 3.92743 \\ \mathrm{H} & 2.27991 & -0.25726 & 2.45345\end{array}$

Cis TS2

E(B3LYP 6-31G(d)) Energy= -1268.71653085 Hartrees Imaginary Freq $=-48.0655 \mathrm{~cm}^{-1}$

$\begin{array}{lccc}\mathrm{O} & -0.56615 & 0.19575 & 2.08875 \\ \mathrm{C} & -0.35684 & 1.56139 & 2.51128 \\ \mathrm{P} & -0.03955 & 0.29905 & 0.44718 \\ \mathrm{C} & 0.30741 & 2.09654 & 1.23452 \\ \mathrm{C} & 1.53379 & 0.26701 & -0.62982 \\ \mathrm{C} & 2.50261 & -0.72194 & -0.39346 \\ \mathrm{C} & 3.72383 & -0.72037 & -1.07133 \\ \mathrm{C} & 3.99845 & 0.26172 & -2.02181 \\ \mathrm{C} & 3.05279 & 1.25889 & -2.26477 \\ \mathrm{C} & 1.84931 & 1.27280 & -1.55867 \\ \mathrm{C} & -1.54958 & 0.72557 & -0.55486 \\ \mathrm{C} & -0.21829 & -1.60441 & 0.35845 \\ \mathrm{C} & -2.73807 & 0.05959 & -0.21340 \\ \mathrm{C} & -3.94068 & 0.36589 & -0.84917 \\ \mathrm{C} & -3.97949 & 1.34192 & -1.84601 \\ \mathrm{C} & -2.80954 & 2.01972 & -2.18716 \\ \mathrm{C} & -1.60936 & 1.72248 & -1.53821 \\ \mathrm{C} & -0.20337 & -2.44441 & 1.48105 \\ \mathrm{C} & -0.32016 & -3.83202 & 1.34238 \\ \mathrm{C} & -0.46205 & -4.40459 & 0.07976 \\ \mathrm{C} & -0.48019 & -3.58085 & -1.04882 \\ \mathrm{C} & -0.35044 & -2.20039 & -0.90722 \\ \mathrm{H} & -1.34204 & 2.02138 & 2.66988 \\ \mathrm{H} & -0.32915 & 2.83448 & 0.73215 \\ \mathrm{H} & 2.30073 & -1.51493 & 0.31929 \\ \mathrm{H} & 4.45455 & -1.49716 & -0.86068 \\ \mathrm{H} & 4.94102 & 0.25591 & -2.56278 \\ \mathrm{H} & 3.25401 & 2.03564 & -2.99839\end{array}$




$\begin{array}{lccc}\mathrm{H} & 1.16091 & 2.08960 & -1.74232 \\ \mathrm{H} & -2.71902 & -0.70230 & 0.55898 \\ \mathrm{H} & -4.84729 & -0.16202 & -0.56535 \\ \mathrm{H} & -4.91454 & 1.57715 & -2.34746 \\ \mathrm{H} & -2.82662 & 2.78801 & -2.95580 \\ \mathrm{H} & -0.72556 & 2.28577 & -1.81134 \\ \mathrm{H} & -0.11187 & -2.00909 & 2.46860 \\ \mathrm{H} & -0.30413 & -4.46244 & 2.22834 \\ \mathrm{H} & -0.55773 & -5.48223 & -0.02710 \\ \mathrm{H} & -0.59201 & -4.01436 & -2.03967 \\ \mathrm{H} & -0.35693 & -1.57712 & -1.79821 \\ \mathrm{C} & 0.42291 & 1.59089 & 3.81861 \\ \mathrm{H} & 0.57757 & 2.62059 & 4.16187 \\ \mathrm{H} & -0.13501 & 1.05406 & 4.59325 \\ \mathrm{H} & 1.40062 & 1.11020 & 3.71167 \\ \mathrm{C} & 1.71682 & 2.66748 & 1.36642 \\ \mathrm{H} & 2.09103 & 3.02829 & 0.40449 \\ \mathrm{H} & 1.72384 & 3.51861 & 2.06079 \\ \mathrm{H} & 2.44103 & 1.93308 & 1.73555\end{array}$

Cis OP2

E(B3LYP 6-31G(d)) Energy=-1268.71970247 Hartrees Number of imaginary frequencies $=0$

$\begin{array}{lccc}\mathrm{O} & 0.516150 & 0.437226 & 2.115750 \\ \mathrm{C} & 1.298525 & -0.661940 & 2.652297 \\ \mathrm{C} & 1.143453 & -1.633597 & 1.479363 \\ \mathrm{P} & 0.110312 & -0.165666 & 0.565521 \\ \mathrm{H} & 0.785684 & -1.018719 & 3.555365 \\ \mathrm{H} & 0.444635 & -2.434071 & 1.745895 \\ \mathrm{C} & -2.498105 & 3.739012 & -0.160417 \\ \mathrm{C} & -2.190951 & 3.352232 & 1.143373 \\ \mathrm{C} & -1.410441 & 2.216159 & 1.379438 \\ \mathrm{C} & -0.918460 & 1.439598 & 0.317778 \\ \mathrm{C} & -1.244485 & 1.843763 & -0.987927 \\ \mathrm{C} & -2.019977 & 2.977943 & -1.228821 \\ \mathrm{H} & -3.102253 & 4.624114 & -0.343624 \\ \mathrm{H} & -2.555758 & 3.936099 & 1.985315 \\ \mathrm{H} & -1.172481 & 1.935200 & 2.397919 \\ \mathrm{H} & -0.885667 & 1.268805 & -1.837439 \\ \mathrm{H} & -2.249689 & 3.267101 & -2.251599\end{array}$




$\begin{array}{lccc}\mathrm{C} & 3.292902 & 0.854489 & -2.691355 \\ \mathrm{C} & 2.372568 & -0.161683 & -2.947911 \\ \mathrm{C} & 1.398508 & -0.488145 & -2.000536 \\ \mathrm{C} & 1.328411 & 0.205941 & -0.785961 \\ \mathrm{C} & 2.250218 & 1.235912 & -0.541761 \\ \mathrm{C} & 3.228264 & 1.553530 & -1.484182 \\ \mathrm{H} & 4.051218 & 1.104656 & -3.428462 \\ \mathrm{H} & 2.407060 & -0.703897 & -3.889483 \\ \mathrm{H} & 0.685183 & -1.274962 & -2.223736 \\ \mathrm{H} & 2.191394 & 1.799405 & 0.385531 \\ \mathrm{H} & 3.934213 & 2.353935 & -1.278133 \\ \mathrm{C} & -3.260127 & -3.111702 & -0.896639 \\ \mathrm{C} & -3.603214 & -1.876153 & -0.350631 \\ \mathrm{C} & -2.608460 & -0.980797 & 0.051092 \\ \mathrm{C} & -1.249676 & -1.311569 & -0.062676 \\ \mathrm{C} & -0.923228 & -2.579699 & -0.577416 \\ \mathrm{C} & -1.912658 & -3.460023 & -1.012626 \\ \mathrm{H} & -4.033844 & -3.802537 & -1.221169 \\ \mathrm{H} & -4.648419 & -1.598995 & -0.240524 \\ \mathrm{H} & -2.898390 & -0.019055 & 0.457681 \\ \mathrm{H} & 0.114134 & -2.890133 & -0.640790 \\ \mathrm{H} & -1.630956 & -4.423840 & -1.428987 \\ \mathrm{C} & 2.693337 & -0.181941 & 3.032387 \\ \mathrm{H} & 3.276395 & -0.997910 & 3.475767 \\ \mathrm{H} & 3.239888 & 0.198842 & 2.164343 \\ \mathrm{H} & 2.621458 & 0.624116 & 3.770377 \\ \mathrm{C} & 2.405450 & -2.251997 & 0.882704 \\ \mathrm{H} & 2.168955 & -2.916164 & 0.043544 \\ \mathrm{H} & 3.106100 & -1.500830 & 0.506764 \\ \mathrm{H} & 2.936139 & -2.859976 & 1.629195 \\ & & & \\ & & & \\ & & & \end{array}$

Cis TS3

E(B3LYP 6-31G(d)) Energy= -1268.69197452 Hartrees Imaginary Freq $=-523.6603 \mathrm{~cm}^{-1}$

$\begin{array}{llll}\mathrm{O} & -0.281472 & -0.002930 & 2.030110 \\ \mathrm{C} & -1.442109 & 1.322416 & 2.334183 \\ \mathrm{C} & -1.813697 & 1.788858 & 1.037746 \\ \mathrm{P} & -0.016269 & 0.086841 & 0.472502 \\ \mathrm{H} & -0.719616 & 1.938929 & 2.868349 \\ \mathrm{H} & -1.362886 & 2.742099 & 0.768789\end{array}$




\begin{tabular}{|c|c|c|c|}
\hline $\mathrm{C}$ & 3.240346 & -3.277048 & 0.236455 \\
\hline $\mathrm{C}$ & 2.730340 & -2.892302 & 1.475964 \\
\hline $\mathrm{C}$ & 1.761572 & -1.888255 & 1.559323 \\
\hline $\mathrm{C}$ & 1.287903 & -1.253700 & 0.401777 \\
\hline $\mathrm{C}$ & 1.809520 & -1.652541 & -0.839739 \\
\hline $\mathrm{C}$ & 2.776612 & -2.652860 & -0.924055 \\
\hline $\mathrm{H}$ & 3.992638 & -4.059096 & 0.172406 \\
\hline $\mathrm{H}$ & 3.083808 & -3.374108 & 2.384213 \\
\hline $\mathrm{H}$ & 1.360421 & -1.589729 & 2.520646 \\
\hline $\mathrm{H}$ & 1.456896 & -1.183097 & -1.754492 \\
\hline $\mathrm{H}$ & 3.166264 & -2.946845 & -1.895328 \\
\hline $\mathrm{C}$ & -3.096927 & -1.981569 & -2.302832 \\
\hline $\mathrm{C}$ & -2.249748 & -1.015264 & -2.847040 \\
\hline $\mathrm{C}$ & -1.320159 & -0.353763 & -2.043020 \\
\hline $\mathrm{C}$ & -1.232014 & -0.648154 & -0.673638 \\
\hline $\mathrm{C}$ & -2.081182 & -1.629548 & -0.134453 \\
\hline $\mathrm{C}$ & -3.005737 & -2.288107 & -0.943721 \\
\hline $\mathrm{H}$ & -3.818748 & -2.493852 & -2.932874 \\
\hline $\mathrm{H}$ & -2.305450 & -0.775393 & -3.905781 \\
\hline $\mathrm{H}$ & -0.661307 & 0.381630 & -2.492268 \\
\hline $\mathrm{H}$ & -2.010104 & -1.879525 & 0.920415 \\
\hline $\mathrm{H}$ & -3.654423 & -3.044718 & -0.510103 \\
\hline $\mathrm{C}$ & 2.514096 & 3.593657 & -1.245683 \\
\hline $\mathrm{C}$ & 3.055420 & 2.685333 & -0.336997 \\
\hline $\mathrm{C}$ & 2.281195 & 1.628691 & 0.145600 \\
\hline $\mathrm{C}$ & 0.941251 & 1.483702 & -0.249273 \\
\hline $\mathrm{C}$ & 0.397525 & 2.423743 & -1.140502 \\
\hline $\mathrm{C}$ & 1.183805 & 3.454541 & -1.650717 \\
\hline $\mathrm{H}$ & 3.119987 & 4.409050 & -1.631565 \\
\hline $\mathrm{H}$ & 4.086023 & 2.788329 & -0.008123 \\
\hline $\mathrm{H}$ & 2.723975 & 0.913123 & 0.830792 \\
\hline $\mathrm{H}$ & -0.648166 & 2.362416 & -1.419546 \\
\hline $\mathrm{H}$ & 0.750082 & 4.163055 & -2.351403 \\
\hline $\mathrm{C}$ & -2.408459 & 0.620352 & 3.258170 \\
\hline $\mathrm{H}$ & -3.114057 & 1.358268 & 3.663751 \\
\hline $\mathrm{H}$ & -2.990875 & -0.139787 & 2.730766 \\
\hline $\mathrm{H}$ & -1.890872 & 0.143137 & 4.094995 \\
\hline $\mathrm{C}$ & -3.211441 & 1.594325 & 0.488710 \\
\hline $\mathrm{H}$ & -3.245096 & 1.826303 & -0.583873 \\
\hline $\mathrm{H}$ & -3.569663 & 0.563482 & 0.590085 \\
\hline $\mathrm{H}$ & -3.961694 & 2.242108 & 0.974181 \\
\hline
\end{tabular}


Cis Butene

E(B3LYP 6-31G(d)) Energy=-157.224774225 Hartrees

Number of imaginary frequencies $=0$

$\begin{array}{lccc}\mathrm{C} & 0.669179 & 0.663806 & 0.000000 \\ \mathrm{C} & -0.669180 & 0.663806 & 0.000000 \\ \mathrm{H} & -1.169189 & 1.633218 & 0.000000 \\ \mathrm{C} & -1.593738 & -0.521617 & 0.000000 \\ \mathrm{H} & -2.251080 & -0.504626 & -0.880084 \\ \mathrm{H} & -1.062036 & -1.477099 & 0.000001 \\ \mathrm{H} & -2.251081 & -0.504625 & 0.880084 \\ \mathrm{H} & 1.169189 & 1.633217 & 0.000000 \\ \mathrm{C} & 1.593738 & -0.521617 & 0.000000 \\ \mathrm{H} & 2.251082 & -0.504624 & 0.880083 \\ \mathrm{H} & 1.062037 & -1.477099 & 0.000001 \\ \mathrm{H} & 2.251080 & -0.504624 & -0.880085\end{array}$

Trans Adduct $\left(\mathrm{Ph}_{3} \mathrm{PCHMe}+\mathrm{MeCHO}\right)$

E(B3LYP 6-31G(d)) Energy=-1268.69431796 Hartrees

Number of imaginary frequencies $=0$

$\begin{array}{lccc}\mathrm{O} & -4.55952 & -0.81976 & 0.47880 \\ \mathrm{C} & -4.25986 & -0.49824 & 1.61002 \\ \mathrm{P} & 0.28811 & 0.02989 & 0.39107 \\ \mathrm{C} & -0.71927 & -0.04824 & 1.76662 \\ \mathrm{C} & 2.12973 & -0.14934 & 0.61822 \\ \mathrm{C} & 2.67095 & -1.42642 & 0.85463 \\ \mathrm{C} & 3.99542 & -1.57943 & 1.26536 \\ \mathrm{C} & 4.80475 & -0.45874 & 1.45861 \\ \mathrm{C} & 4.27592 & 0.81629 & 1.24776 \\ \mathrm{C} & 2.95068 & 0.96989 & 0.84081 \\ \mathrm{C} & 0.06358 & 1.64354 & -0.44955 \\ \mathrm{C} & -0.14134 & -1.27981 & -0.82838 \\ \mathrm{C} & -1.01324 & 2.46861 & -0.09747 \\ \mathrm{C} & -1.22351 & 3.67634 & -0.76326 \\ \mathrm{C} & -0.37535 & 4.06271 & -1.80235 \\ \mathrm{C} & 0.68646 & 3.23595 & -2.17450 \\ \mathrm{C} & 0.90521 & 2.03326 & -1.50293 \\ \mathrm{C} & -1.46738 & -1.73533 & -0.86661 \\ \mathrm{C} & -1.86457 & -2.66777 & -1.82547\end{array}$




$\begin{array}{lccc}\mathrm{C} & -0.94800 & -3.14338 & -2.76441 \\ \mathrm{C} & 0.36984 & -2.68138 & -2.74422 \\ \mathrm{C} & 0.77244 & -1.75414 & -1.78274 \\ \mathrm{H} & -3.69975 & -1.19611 & 2.26658 \\ \mathrm{H} & -0.69009 & 0.88843 & 2.32794 \\ \mathrm{H} & 2.05596 & -2.31047 & 0.70961 \\ \mathrm{H} & 4.39458 & -2.57639 & 1.43394 \\ \mathrm{H} & 5.83706 & -0.57734 & 1.77674 \\ \mathrm{H} & 4.89649 & 1.69572 & 1.40043 \\ \mathrm{H} & 2.55438 & 1.97022 & 0.69377 \\ \mathrm{H} & -1.69024 & 2.14470 & 0.68566 \\ \mathrm{H} & -2.05803 & 4.31048 & -0.47613 \\ \mathrm{H} & -0.54580 & 4.99939 & -2.32645 \\ \mathrm{H} & 1.34508 & 3.52564 & -2.98887 \\ \mathrm{H} & 1.73746 & 1.40081 & -1.79779 \\ \mathrm{H} & -2.18859 & -1.34760 & -0.15584 \\ \mathrm{H} & -2.89539 & -3.01059 & -1.83780 \\ \mathrm{H} & -1.25922 & -3.86626 & -3.51418 \\ \mathrm{H} & 1.08695 & -3.04339 & -3.47637 \\ \mathrm{H} & 1.80154 & -1.40864 & -1.77291 \\ \mathrm{C} & -4.60808 & 0.82511 & 2.24200 \\ \mathrm{H} & -3.68213 & 1.32411 & 2.55353 \\ \mathrm{H} & -5.15877 & 1.45380 & 1.53828 \\ \mathrm{H} & -5.20677 & 0.66263 & 3.14814 \\ \mathrm{C} & -0.71680 & -1.30543 & 2.62184 \\ \mathrm{H} & -1.54995 & -1.28194 & 3.33771 \\ \mathrm{H} & -0.85479 & -2.21045 & 2.01548 \\ \mathrm{H} & 0.19914 & -1.46143 & 3.21832\end{array}$

Trans TS1

E(B3LYP 6-31G(d)) Energy= -1268.68772564 Hartrees Imaginary Freq $=-150.3451 \mathrm{~cm}^{-1}$

$\begin{array}{lccc}\mathrm{O} & -3.307073 & -0.258688 & 1.219290 \\ \mathrm{C} & -2.574068 & -0.456442 & 2.222344 \\ \mathrm{P} & 0.148730 & 0.023342 & 0.257844 \\ \mathrm{C} & -0.501554 & -0.059003 & 1.900787 \\ \mathrm{C} & 1.983630 & 0.217792 & 0.275295 \\ \mathrm{C} & 2.810469 & -0.901240 & 0.484462 \\ \mathrm{C} & 4.178720 & -0.744901 & 0.709759 \\ \mathrm{C} & 4.744500 & 0.529749 & 0.744553\end{array}$




\begin{tabular}{|c|c|c|c|}
\hline $\mathrm{C}$ & 3.930387 & 1.650151 & 0.566459 \\
\hline $\mathrm{C}$ & 2.562484 & 1.497937 & 0.344612 \\
\hline $\mathrm{C}$ & -0.547539 & 1.439431 & -0.657068 \\
\hline $\mathrm{C}$ & -0.228761 & -1.477981 & -0.721431 \\
\hline $\mathrm{C}$ & -1.843693 & 1.881918 & -0.346780 \\
\hline $\mathrm{C}$ & -2.408532 & 2.923802 & -1.086793 \\
\hline $\mathrm{C}$ & -1.703757 & 3.518466 & -2.132801 \\
\hline $\mathrm{C}$ & -0.427457 & 3.055954 & -2.463152 \\
\hline $\mathrm{C}$ & 0.146906 & 2.016017 & -1.735370 \\
\hline $\mathrm{C}$ & -1.510476 & -2.042527 & -0.593394 \\
\hline $\mathrm{C}$ & -1.857571 & -3.143910 & -1.378368 \\
\hline $\mathrm{C}$ & -0.951191 & -3.680237 & -2.293711 \\
\hline $\mathrm{C}$ & 0.309515 & -3.099806 & -2.444741 \\
\hline $\mathrm{C}$ & 0.668962 & -1.998872 & -1.667923 \\
\hline $\mathrm{H}$ & -2.303070 & -1.499915 & 2.490154 \\
\hline $\mathrm{H}$ & -0.421805 & 0.956087 & 2.305091 \\
\hline $\mathrm{H}$ & 2.388548 & -1.901002 & 0.467131 \\
\hline $\mathrm{H}$ & 4.800536 & -1.623199 & 0.860588 \\
\hline $\mathrm{H}$ & 5.810185 & 0.650414 & 0.918441 \\
\hline $\mathrm{H}$ & 4.359426 & 2.647870 & 0.602780 \\
\hline $\mathrm{H}$ & 1.943902 & 2.381450 & 0.224105 \\
\hline $\mathrm{H}$ & -2.436387 & 1.375764 & 0.415179 \\
\hline $\mathrm{H}$ & -3.412701 & 3.260755 & -0.844552 \\
\hline $\mathrm{H}$ & -2.150552 & 4.330454 & -2.700690 \\
\hline $\mathrm{H}$ & 0.119928 & 3.500874 & -3.289735 \\
\hline $\mathrm{H}$ & 1.135295 & 1.659032 & -2.008039 \\
\hline $\mathrm{H}$ & -2.251736 & -1.590366 & 0.068184 \\
\hline $\mathrm{H}$ & -2.849100 & -3.576350 & -1.275448 \\
\hline $\mathrm{H}$ & -1.229563 & -4.539529 & -2.898411 \\
\hline $\mathrm{H}$ & 1.014106 & -3.500237 & -3.168703 \\
\hline $\mathrm{H}$ & 1.647655 & -1.550700 & -1.803396 \\
\hline $\mathrm{C}$ & -2.752422 & 0.442403 & 3.446985 \\
\hline $\mathrm{H}$ & -1.975168 & 0.307947 & 4.206820 \\
\hline $\mathrm{H}$ & -2.790244 & 1.494480 & 3.145752 \\
\hline $\mathrm{H}$ & -3.720132 & 0.196191 & 3.905265 \\
\hline $\mathrm{C}$ & 0.207985 & -1.091313 & 2.775910 \\
\hline $\mathrm{H}$ & -0.278145 & -1.134456 & 3.758066 \\
\hline $\mathrm{H}$ & 0.130328 & -2.098931 & 2.346606 \\
\hline $\mathrm{H}$ & 1.275104 & -0.891585 & 2.955015 \\
\hline
\end{tabular}

Trans OP1 
E(B3LYP 6-31G(d)) Energy=-1268.72671508 Hartrees Number of imaginary frequencies $=0$

\begin{tabular}{|c|c|c|c|}
\hline $\mathrm{O}$ & -0.424992 & -1.902117 & 1.203218 \\
\hline $\mathrm{C}$ & -0.126862 & -1.526102 & 2.539365 \\
\hline $\mathrm{P}$ & -0.063448 & -0.279505 & 0.427863 \\
\hline $\mathrm{C}$ & 0.124513 & -0.030103 & 2.313590 \\
\hline $\mathrm{C}$ & 0.362825 & 1.578902 & 0.075698 \\
\hline $\mathrm{C}$ & 1.625313 & 2.033613 & -0.334334 \\
\hline $\mathrm{C}$ & 1.938230 & 3.397405 & -0.364842 \\
\hline $\mathrm{C}$ & 0.994792 & 4.345459 & 0.025567 \\
\hline $\mathrm{C}$ & -0.264739 & 3.917058 & 0.452290 \\
\hline $\mathrm{C}$ & -0.567790 & 2.556135 & 0.479743 \\
\hline $\mathrm{C}$ & -1.686474 & -0.293740 & -0.500628 \\
\hline $\mathrm{C}$ & 1.273566 & -1.035218 & -0.609828 \\
\hline $\mathrm{C}$ & -2.678834 & -1.239316 & -0.192282 \\
\hline $\mathrm{C}$ & -3.871496 & -1.287470 & -0.91614 \\
\hline $\mathrm{C}$ & -4.090159 & -0.408557 & -1.97616 \\
\hline $\mathrm{C}$ & -3.106536 & 0.524340 & -2.30665 \\
\hline $\mathrm{C}$ & -1.922455 & 0.586675 & -1.572231 \\
\hline $\mathrm{C}$ & 2.152189 & -1.983560 & -0.06701 \\
\hline $\mathrm{C}$ & 3.154924 & -2.555473 & -0.85132 \\
\hline $\mathrm{C}$ & 3.271651 & -2.216710 & -2.19973 \\
\hline $\mathrm{C}$ & 2.384761 & -1.294986 & -2.75785 \\
\hline $\mathrm{C}$ & 1.402542 & -0.699839 & -1.96566 \\
\hline $\mathrm{H}$ & 0.800353 & -2.020604 & 2.883214 \\
\hline $\mathrm{H}$ & -0.733974 & 0.575527 & 2.635898 \\
\hline $\mathrm{H}$ & 2.387425 & 1.322204 & -0.63577 \\
\hline $\mathrm{H}$ & 2.925236 & 3.713750 & -0.694207 \\
\hline $\mathrm{H}$ & 1.236052 & 5.405041 & 0.001867 \\
\hline $\mathrm{H}$ & -1.012116 & 4.643572 & 0.762564 \\
\hline $\mathrm{H}$ & -1.559823 & 2.251966 & 0.80811 \\
\hline $\mathrm{H}$ & -2.490288 & -1.946547 & 0.60387 \\
\hline $\mathrm{H}$ & -4.626963 & -2.023116 & -0.65229 \\
\hline $\mathrm{H}$ & -5.016930 & -0.450907 & -2.54272 \\
\hline $\mathrm{H}$ & -3.260065 & 1.212039 & -3.13421 \\
\hline $\mathrm{H}$ & -1.186961 & 1.337460 & -1.835545 \\
\hline $\mathrm{H}$ & 2.040707 & -2.295139 & 0.96479 \\
\hline $\mathrm{H}$ & 3.833641 & -3.280393 & -0.409667 \\
\hline $\mathrm{H}$ & 4.042888 & -2.673976 & -2.81398 \\
\hline $\mathrm{H}$ & 2.456874 & -1.034536 & -3.81054 \\
\hline $\mathrm{H}$ & 0.729274 & 0.023713 & -2.41348 \\
\hline
\end{tabular}




$\begin{array}{llll}\mathrm{C} & -1.250238 & -1.881367 & 3.510030 \\ \mathrm{H} & -0.993205 & -1.585170 & 4.535047 \\ \mathrm{H} & -2.182175 & -1.377216 & 3.227501 \\ \mathrm{H} & -1.431727 & -2.961587 & 3.503499 \\ \mathrm{C} & 1.409990 & 0.576907 & 2.868900 \\ \mathrm{H} & 1.400090 & 0.524059 & 3.965345 \\ \mathrm{H} & 2.298042 & 0.038112 & 2.518985 \\ \mathrm{H} & 1.517014 & 1.626950 & 2.585094\end{array}$

Trans TS2

E(B3LYP 6-31G(d)) Energy= -1268.71808316 Hartrees Imaginary Freq $=-48.9687 \mathrm{~cm}^{-1}$

$\begin{array}{lccc}\mathrm{O} & -0.74152 & -0.12398 & 2.06961 \\ \mathrm{C} & -1.14015 & 1.20407 & 2.48616 \\ \mathrm{P} & -0.09147 & 0.24438 & 0.51269 \\ \mathrm{C} & -0.44640 & 2.00235 & 1.38277 \\ \mathrm{C} & 1.53170 & 0.79466 & -0.31930 \\ \mathrm{C} & 2.72959 & 0.12317 & -0.02740 \\ \mathrm{C} & 3.95297 & 0.56577 & -0.53631 \\ \mathrm{C} & 4.00526 & 1.68221 & -1.36896 \\ \mathrm{C} & 2.82719 & 2.37206 & -1.66049 \\ \mathrm{C} & 1.61351 & 1.94680 & -1.11979 \\ \mathrm{C} & -1.50017 & 0.23486 & -0.70659 \\ \mathrm{C} & 0.37063 & -1.60754 & 0.36228 \\ \mathrm{C} & -2.42447 & -0.81833 & -0.60657 \\ \mathrm{C} & -3.55551 & -0.86087 & -1.42105 \\ \mathrm{C} & -3.78279 & 0.14583 & -2.36077 \\ \mathrm{C} & -2.87850 & 1.20222 & -2.46532 \\ \mathrm{C} & -1.75593 & 1.25186 & -1.63643 \\ \mathrm{C} & 0.52145 & -2.46486 & 1.46177 \\ \mathrm{C} & 0.88523 & -3.80450 & 1.28478 \\ \mathrm{C} & 1.09713 & -4.31432 & 0.00513 \\ \mathrm{C} & 0.94990 & -3.47333 & -1.10100 \\ \mathrm{C} & 0.60007 & -2.13634 & -0.91945 \\ \mathrm{H} & -0.69284 & 1.39272 & 3.47201 \\ \mathrm{H} & -1.13349 & 2.62426 & 0.79541 \\ \mathrm{H} & 2.71226 & -0.76321 & 0.59757 \\ \mathrm{H} & 4.86342 & 0.02702 & -0.28635 \\ \mathrm{H} & 4.95365 & 2.01864 & -1.77949 \\ \mathrm{H} & 2.85232 & 3.25087 & -2.30002 \\ & & & \\ \mathrm{C} & & & \\ \mathrm{C} & & & \end{array}$




$\begin{array}{lccc}\mathrm{H} & 0.72816 & 2.53825 & -1.32393 \\ \mathrm{H} & -2.25720 & -1.60812 & 0.11839 \\ \mathrm{H} & -4.25735 & -1.68474 & -1.32185 \\ \mathrm{H} & -4.66015 & 0.11054 & -3.00112 \\ \mathrm{H} & -3.04651 & 1.99687 & -3.18758 \\ \mathrm{H} & -1.08749 & 2.09905 & -1.72768 \\ \mathrm{H} & 0.34221 & -2.08458 & 2.45991 \\ \mathrm{H} & 0.99737 & -4.44866 & 2.15373 \\ \mathrm{H} & 1.37465 & -5.35645 & -0.13205 \\ \mathrm{H} & 1.11115 & -3.85809 & -2.10508 \\ \mathrm{H} & 0.50043 & -1.49629 & -1.79284 \\ \mathrm{C} & -2.65625 & 1.32643 & 2.58688 \\ \mathrm{H} & -3.13382 & 1.16456 & 1.61542 \\ \mathrm{H} & -3.05432 & 0.58825 & 3.29164 \\ \mathrm{H} & -2.93276 & 2.32524 & 2.94624 \\ \mathrm{C} & 0.72773 & 2.84331 & 1.88034 \\ \mathrm{H} & 0.37154 & 3.62685 & 2.56361 \\ \mathrm{H} & 1.45709 & 2.23489 & 2.42939 \\ \mathrm{H} & 1.26276 & 3.33516 & 1.06338\end{array}$

Trans OP2

E(B3LYP 6-31G(d)) Energy=-1268.72671569 Hartrees Number of imaginary frequencies $=0$

$\begin{array}{lccc}\mathrm{O} & -0.422363 & 1.902724 & 1.203310 \\ \mathrm{C} & -0.124605 & 1.526227 & 2.539440 \\ \mathrm{C} & 0.125662 & 0.030032 & 2.313448 \\ \mathrm{P} & -0.062856 & 0.279748 & 0.427883 \\ \mathrm{H} & 0.802915 & 2.020021 & 2.883468 \\ \mathrm{H} & -0.732891 & -0.575199 & 2.636282 \\ \mathrm{C} & -4.089424 & 0.414767 & -1.976216 \\ \mathrm{C} & -3.869443 & 1.293409 & -0.916239 \\ \mathrm{C} & -2.676892 & 1.243450 & -0.192323 \\ \mathrm{C} & -1.685968 & 0.296318 & -0.500549 \\ \mathrm{C} & -1.923286 & -0.583802 & -1.572097 \\ \mathrm{C} & -3.107237 & -0.519686 & -2.306572 \\ \mathrm{H} & -5.016100 & 0.458521 & -2.542823 \\ \mathrm{H} & -4.623765 & 2.030261 & -0.652476 \\ \mathrm{H} & -2.487264 & 1.950446 & 0.603779 \\ \mathrm{H} & -1.188958 & -1.335748 & -1.835324 \\ \mathrm{H} & -3.261778 & -1.207210 & -3.134090\end{array}$




$\begin{array}{lccc}\mathrm{C} & 3.274974 & 2.211927 & -2.199747 \\ \mathrm{C} & 2.387304 & 1.290764 & -2.757577 \\ \mathrm{C} & 1.404320 & 0.697051 & -1.965273 \\ \mathrm{C} & 1.275389 & 1.033308 & -0.609627 \\ \mathrm{C} & 2.154800 & 1.981090 & -0.067145 \\ \mathrm{C} & 3.158285 & 2.551559 & -0.851562 \\ \mathrm{H} & 4.046798 & 2.668094 & -2.814084 \\ \mathrm{H} & 2.459411 & 1.029661 & -3.810104 \\ \mathrm{H} & 0.730445 & -0.026136 & -2.412783 \\ \mathrm{H} & 2.043320 & 2.293404 & 0.964434 \\ \mathrm{H} & 3.837636 & 3.276040 & -0.410162 \\ \mathrm{C} & 0.987073 & -4.347264 & 0.025846 \\ \mathrm{C} & -0.271494 & -3.916510 & 0.453059 \\ \mathrm{C} & -0.572095 & -2.555047 & 0.480356 \\ \mathrm{C} & 0.360101 & -1.579579 & 0.075689 \\ \mathrm{C} & 1.621580 & -2.036638 & -0.334820 \\ \mathrm{C} & 1.932036 & -3.401001 & -0.365204 \\ \mathrm{H} & 1.226405 & -5.407287 & 0.002288 \\ \mathrm{H} & -1.020016 & -4.641634 & 0.763829 \\ \mathrm{H} & -1.563443 & -2.249033 & 0.809084 \\ \mathrm{H} & 2.384832 & -1.326627 & -0.636677 \\ \mathrm{H} & 2.918322 & -3.719188 & -0.694950 \\ \mathrm{C} & 1.411239 & -0.577671 & 2.867798 \\ \mathrm{H} & 1.517650 & -1.627684 & 2.583655 \\ \mathrm{H} & 2.299286 & -0.039157 & 2.517438 \\ \mathrm{H} & 1.402091 & -0.525102 & 3.964262 \\ \mathrm{C} & -1.247863 & 1.882107 & 3.510029 \\ \mathrm{H} & -1.991183 & 1.585570 & 4.535035 \\ \mathrm{H} & -2.180107 & 1.378650 & 3.227280 \\ \mathrm{H} & & & 3.503637 \\ & & \end{array}$

Trans TS3

E(B3LYP 6-31G(d)) Energy= -1268.69383573 Hartrees Imaginary Freq $=-516.3488 \mathrm{~cm}^{-1}$

$\begin{array}{llll}\mathrm{O} & 0.098230 & -0.169149 & 2.052666 \\ \mathrm{C} & 1.097581 & -1.623153 & 2.339059 \\ \mathrm{C} & 1.529608 & -2.037392 & 1.047157 \\ \mathrm{P} & 0.027130 & -0.072200 & 0.475260 \\ \mathrm{H} & 1.834128 & -1.110755 & 2.959893 \\ \mathrm{H} & 1.017419 & -2.920341 & 0.663514\end{array}$




\begin{tabular}{|c|c|c|c|}
\hline $\mathrm{C}$ & -2.848958 & 3.622058 & 0.243727 \\
\hline $\mathrm{C}$ & -2.532739 & 3.066731 & 1.483125 \\
\hline $\mathrm{C}$ & -1.679159 & 1.963066 & 1.564585 \\
\hline $\mathrm{C}$ & -1.129199 & 1.397403 & 0.404990 \\
\hline $\mathrm{C}$ & -1.455574 & 1.967868 & -0.836002 \\
\hline $\mathrm{C}$ & -2.306694 & 3.068635 & -0.918621 \\
\hline $\mathrm{H}$ & -3.511873 & 4.481304 & 0.181520 \\
\hline $\mathrm{H}$ & -2.948875 & 3.491850 & 2.393124 \\
\hline $\mathrm{H}$ & -1.429018 & 1.531777 & 2.526623 \\
\hline $\mathrm{H}$ & -1.041093 & 1.553631 & -1.751334 \\
\hline $\mathrm{H}$ & -2.545404 & 3.495174 & -1.889535 \\
\hline $\mathrm{C}$ & 3.663520 & 1.941407 & -1.565342 \\
\hline $\mathrm{C}$ & 2.785759 & 1.195350 & -2.352352 \\
\hline $\mathrm{C}$ & 1.689459 & 0.551669 & -1.774874 \\
\hline $\mathrm{C}$ & 1.464414 & 0.640482 & -0.392765 \\
\hline $\mathrm{C}$ & 2.348802 & 1.398761 & 0.392492 \\
\hline $\mathrm{C}$ & 3.438106 & 2.043001 & -0.190798 \\
\hline $\mathrm{H}$ & 4.514422 & 2.441925 & -2.019139 \\
\hline $\mathrm{H}$ & 2.947751 & 1.115351 & -3.424185 \\
\hline $\mathrm{H}$ & 1.010900 & -0.008824 & -2.409249 \\
\hline $\mathrm{H}$ & 2.177390 & 1.482450 & 1.462032 \\
\hline $\mathrm{H}$ & 4.111988 & 2.626557 & 0.430947 \\
\hline $\mathrm{C}$ & -2.512863 & -3.069169 & -2.016288 \\
\hline $\mathrm{C}$ & -3.103042 & -2.241187 & -1.062636 \\
\hline $\mathrm{C}$ & -2.325159 & -1.331437 & -0.343194 \\
\hline $\mathrm{C}$ & -0.937315 & -1.262048 & -0.544855 \\
\hline $\mathrm{C}$ & -0.348954 & -2.124136 & -1.484907 \\
\hline $\mathrm{C}$ & -1.132810 & -3.002023 & -2.229690 \\
\hline $\mathrm{H}$ & -3.120070 & -3.767782 & -2.585561 \\
\hline $\mathrm{H}$ & -4.173545 & -2.290962 & -0.882260 \\
\hline $\mathrm{H}$ & -2.801924 & -0.671757 & 0.374819 \\
\hline $\mathrm{H}$ & 0.727063 & -2.123008 & -1.617418 \\
\hline $\mathrm{H}$ & -0.662214 & -3.649940 & -2.964403 \\
\hline $\mathrm{C}$ & 0.118314 & -2.456388 & 3.132199 \\
\hline $\mathrm{H}$ & 0.653515 & -3.308921 & 3.571583 \\
\hline $\mathrm{H}$ & -0.348897 & -1.888107 & 3.941629 \\
\hline $\mathrm{H}$ & -0.668671 & -2.854685 & 2.483342 \\
\hline $\mathrm{C}$ & 2.991566 & -1.947697 & 0.661536 \\
\hline $\mathrm{H}$ & 3.131085 & -1.858660 & -0.425158 \\
\hline $\mathrm{H}$ & 3.474737 & -1.070538 & 1.108307 \\
\hline $\mathrm{H}$ & 3.570414 & -2.830343 & 0.981413 \\
\hline
\end{tabular}


Trans butene

E(B3LYP 6-31G(d)) Energy=-157.226910330 Hartrees

Number of imaginary frequencies $=0$

$\begin{array}{lccc}\mathrm{C} & -0.538103 & -0.395365 & 0.000000 \\ \mathrm{C} & 0.538103 & 0.395364 & 0.000000 \\ \mathrm{H} & 0.392436 & 1.477727 & 0.000000 \\ \mathrm{H} & -0.392437 & -1.477727 & 0.000001 \\ \mathrm{C} & 1.963509 & -0.079223 & 0.000000 \\ \mathrm{H} & 2.507882 & 0.289068 & -0.880441 \\ \mathrm{H} & 2.507884 & 0.289072 & 0.880437 \\ \mathrm{H} & 2.022776 & -1.173126 & 0.000001 \\ \mathrm{C} & -1.963509 & 0.079223 & 0.000000 \\ \mathrm{H} & -2.507885 & -0.289069 & -0.880438 \\ \mathrm{H} & -2.507883 & -0.289070 & 0.880438 \\ \mathrm{H} & -2.022777 & 1.173126 & 0.000001\end{array}$

Ph3PO

E(B3LYP 6-31G(d)) Energy=-1111.55122457 Hartrees

Number of imaginary frequencies $=0$

$\begin{array}{lccc}\mathrm{O} & -0.00139 & 0.00134 & 2.41787 \\ \mathrm{P} & -0.00097 & 0.00149 & 0.91434 \\ \mathrm{C} & 1.04821 & -4.20452 & -0.71615 \\ \mathrm{C} & 1.31947 & -3.09187 & -1.51486 \\ \mathrm{C} & 1.02415 & -1.80902 & -1.05073 \\ \mathrm{C} & 0.44590 & -1.63162 & 0.21448 \\ \mathrm{C} & 0.18853 & -2.75301 & 1.01703 \\ \mathrm{C} & 0.48715 & -4.03343 & 0.55125 \\ \mathrm{H} & 1.28268 & -5.20242 & -1.07736 \\ \mathrm{H} & 1.77007 & -3.22110 & -2.49538 \\ \mathrm{H} & 1.26340 & -0.94773 & -1.66850 \\ \mathrm{H} & -0.22812 & -2.60771 & 2.00932 \\ \mathrm{H} & 0.28749 & -4.89716 & 1.17982 \\ \mathrm{C} & 3.12348 & 3.00666 & -0.71502 \\ \mathrm{C} & 2.01894 & 2.69508 & -1.51026 \\ \mathrm{C} & 1.05302 & 1.80043 & -1.04652 \\ \mathrm{C} & 1.19128 & 1.20390 & 0.21507 \\ \mathrm{C} & 2.29653 & 1.53210 & 1.01434 \\ \mathrm{C} & 3.25867 & 2.42820 & 0.54890\end{array}$




$\begin{array}{lccc}\mathrm{H} & 3.87235 & 3.70667 & -1.07620 \\ \mathrm{H} & 1.90363 & 3.15535 & -2.48803 \\ \mathrm{H} & 0.18393 & 1.58413 & -1.66184 \\ \mathrm{H} & 2.38170 & 1.09302 & 2.00397 \\ \mathrm{H} & 4.11069 & 2.67953 & 1.17489 \\ \mathrm{C} & -4.16945 & 1.19284 & -0.71421 \\ \mathrm{C} & -3.34231 & 0.40017 & -1.51235 \\ \mathrm{C} & -2.08277 & 0.01616 & -1.04917 \\ \mathrm{C} & -1.63856 & 0.43121 & 0.21455 \\ \mathrm{C} & -2.48006 & 1.21663 & 1.01638 \\ \mathrm{C} & -3.73905 & 1.59656 & 0.55156 \\ \mathrm{H} & -5.15167 & 1.48716 & -1.07458 \\ \mathrm{H} & -3.68092 & 0.07194 & -2.49151 \\ \mathrm{H} & -1.45683 & -0.62290 & -1.66596 \\ \mathrm{H} & -2.14486 & 1.50706 & 2.00768 \\ \mathrm{H} & -4.38672 & 2.20236 & 1.17967\end{array}$

\section{Stabilized Ylides $\left(\mathrm{Me}_{3} \mathrm{PCHCOOMe}+\mathrm{PhCHO}\right)$}

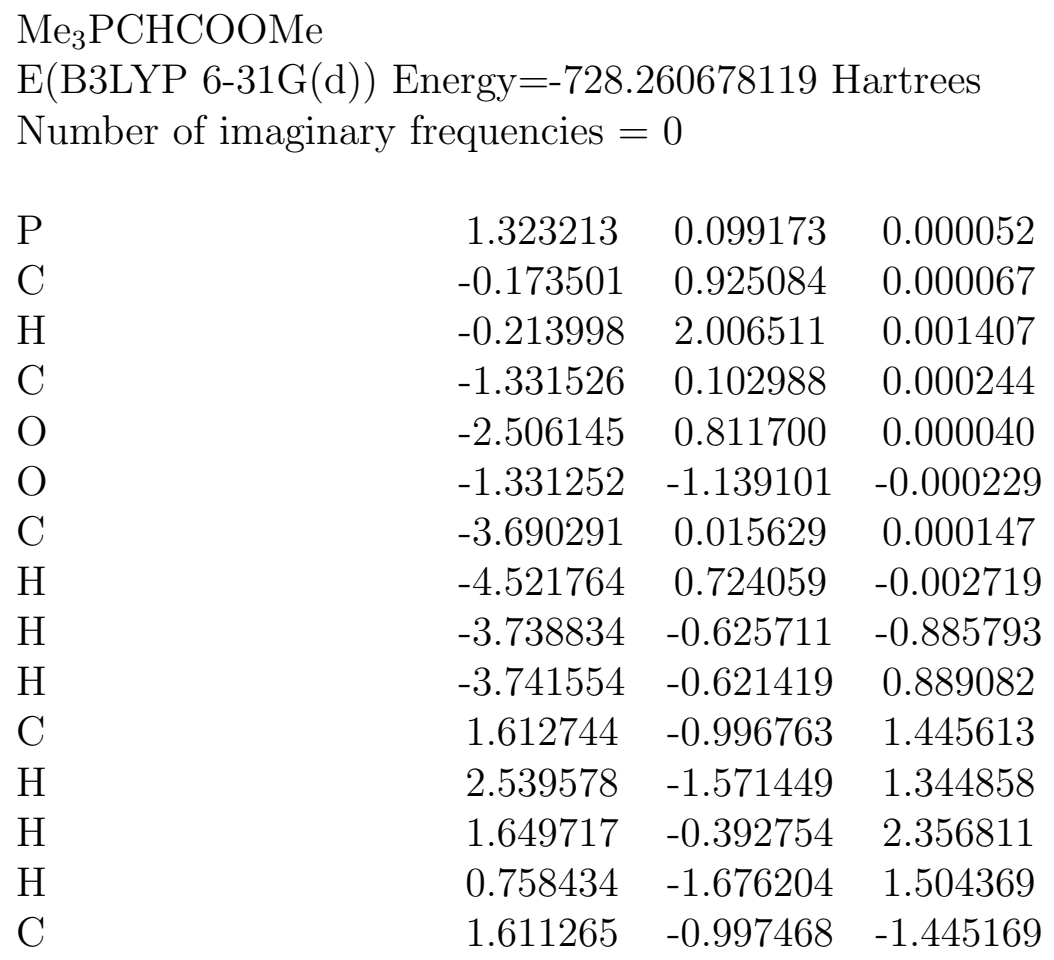




$\begin{array}{cccc}\mathrm{H} & 2.537932 & -1.572494 & -1.344724 \\ \mathrm{H} & 0.756672 & -1.676610 & -1.503061 \\ \mathrm{H} & 1.647929 & -0.393883 & -2.356640 \\ \mathrm{C} & 2.686432 & 1.319530 & -0.000862 \\ \mathrm{H} & 3.655889 & 0.812656 & 0.001329 \\ \mathrm{H} & 2.616164 & 1.950672 & -0.891265 \\ \mathrm{H} & 2.614066 & 1.954238 & 0.886835\end{array}$

\section{$\mathrm{PhCHO}$}

E(B3LYP 6-31G(d)) Energy=-345.573455692 Hartrees Number of imaginary frequencies $=0$

$\begin{array}{lccc}\mathrm{C} & 0.592145 & -2.150954 & 0.000000 \\ \mathrm{C} & 1.630339 & -1.216571 & 0.000000 \\ \mathrm{C} & 1.333323 & 0.145608 & 0.000000 \\ \mathrm{C} & 0.000000 & 0.575208 & 0.000000 \\ \mathrm{C} & -1.039168 & -0.368003 & 0.000000 \\ \mathrm{C} & -0.742102 & -1.726587 & 0.000000 \\ \mathrm{H} & 0.821246 & -3.213358 & 0.000000 \\ \mathrm{H} & 2.664247 & -1.550104 & 0.000000 \\ \mathrm{H} & 2.133766 & 0.882781 & 0.000000 \\ \mathrm{H} & -2.064294 & -0.010180 & 0.000000 \\ \mathrm{H} & -1.544252 & -2.459452 & 0.000000 \\ \mathrm{C} & -0.305966 & 2.023605 & 0.000000 \\ \mathrm{O} & -1.426439 & 2.496397 & 0.000000 \\ \mathrm{H} & 0.589368 & 2.685305 & 0.000000\end{array}$

Cis Adduct $\left(\mathrm{Me}_{3} \mathrm{PCHCOOMe}+\mathrm{PhCHO}\right)$

E(B3LYP 6-31G(d)) Energy=-1073.84247798 Hartrees Number of imaginary frequencies $=0$

$\begin{array}{lccc}\mathrm{O} & -0.55704 & -2.60859 & -0.93314 \\ \mathrm{C} & -1.03468 & -1.51506 & -1.20206 \\ \mathrm{P} & 2.49794 & -0.63214 & 0.14897 \\ \mathrm{C} & 1.60577 & 0.50315 & -0.77927 \\ \mathrm{C} & -2.17923 & -0.90407 & -0.49567 \\ \mathrm{C} & -2.92644 & -1.65125 & 0.42813 \\ \mathrm{C} & -4.00793 & -1.06710 & 1.07871 \\ \mathrm{C} & -4.34152 & 0.26743 & 0.81831 \\ \mathrm{C} & -3.59396 & 1.01782 & -0.09240\end{array}$




$\begin{array}{lccc}\mathrm{C} & -2.51585 & 0.43213 & -0.75441 \\ \mathrm{H} & -0.61926 & -0.90535 & -2.02628 \\ \mathrm{H} & 1.48698 & 0.36172 & -1.84567 \\ \mathrm{H} & -2.64452 & -2.68359 & 0.61245 \\ \mathrm{H} & -4.59375 & -1.64438 & 1.78884 \\ \mathrm{H} & -5.18545 & 0.72276 & 1.33007 \\ \mathrm{H} & -3.85066 & 2.05602 & -0.28330 \\ \mathrm{H} & -1.90755 & 1.01468 & -1.44186 \\ \mathrm{C} & 2.88401 & -2.07902 & -0.89591 \\ \mathrm{H} & 3.49846 & -2.79582 & -0.34302 \\ \mathrm{H} & 1.94552 & -2.55785 & -1.19253 \\ \mathrm{H} & 3.42868 & -1.75751 & -1.78855 \\ \mathrm{C} & 4.09297 & -0.00674 & 0.81676 \\ \mathrm{H} & 3.87135 & 0.89778 & 1.39011 \\ \mathrm{H} & 4.58353 & -0.74276 & 1.46279 \\ \mathrm{H} & 4.75619 & 0.25411 & -0.01317 \\ \mathrm{C} & 1.61156 & -1.26908 & 1.62103 \\ \mathrm{H} & 2.26758 & -1.88394 & 2.24597 \\ \mathrm{H} & 1.26036 & -0.40385 & 2.18895 \\ \mathrm{H} & 0.75489 & -1.86194 & 1.29029 \\ \mathrm{C} & 1.15072 & 1.66643 & -0.10764 \\ \mathrm{O} & 0.42106 & 2.51189 & -0.91943 \\ \mathrm{O} & 1.34484 & 1.94307 & 1.08488 \\ \mathrm{C} & -0.03332 & 3.70529 & -0.28113 \\ \mathrm{H} & -0.54129 & 4.28143 & -1.05824 \\ \mathrm{H} & -0.72665 & 3.47939 & 0.53567 \\ \mathrm{H} & 0.80305 & 4.28064 & 0.12762\end{array}$

Cis TS1

E(B3LYP 6-31G(d)) Energy= -1073.81204349 Hartrees Imaginary Freq $=-251.4229 \mathrm{~cm}^{-1}$

$\begin{array}{lccc}\mathrm{O} & 0.679961 & -2.049523 & -0.844734 \\ \mathrm{C} & -0.053043 & -1.027455 & -1.178520 \\ \mathrm{P} & 2.334764 & -0.268345 & 0.104789 \\ \mathrm{C} & 0.901160 & 0.458444 & -0.746093 \\ \mathrm{C} & -1.435698 & -0.908614 & -0.536970 \\ \mathrm{C} & -1.758613 & -1.712456 & 0.559735 \\ \mathrm{C} & -3.024514 & -1.647648 & 1.143229 \\ \mathrm{C} & -3.991346 & -0.781877 & 0.627656 \\ \mathrm{C} & -3.686225 & 0.009050 & -0.483423\end{array}$




$\begin{array}{lccc}\mathrm{C} & -2.418664 & -0.060342 & -1.063471 \\ \mathrm{H} & -0.161842 & -0.814475 & -2.267479 \\ \mathrm{H} & 1.233303 & 0.865054 & -1.704330 \\ \mathrm{H} & -1.005397 & -2.406063 & 0.919506 \\ \mathrm{H} & -3.261894 & -2.280477 & 1.995443 \\ \mathrm{H} & -4.979923 & -0.733682 & 1.077724 \\ \mathrm{H} & -4.441064 & 0.668389 & -0.906193 \\ \mathrm{H} & -2.188381 & 0.547709 & -1.935701 \\ \mathrm{C} & 3.412282 & -1.166259 & -1.060432 \\ \mathrm{H} & 4.280431 & -1.566328 & -0.526121 \\ \mathrm{H} & 2.808388 & -1.965553 & -1.492512 \\ \mathrm{H} & 3.761239 & -0.477328 & -1.836734 \\ \mathrm{C} & 3.373056 & 1.123877 & 0.708177 \\ \mathrm{H} & 2.797922 & 1.716788 & 1.423158 \\ \mathrm{H} & 4.274947 & 0.739938 & 1.196709 \\ \mathrm{H} & 3.671714 & 1.763612 & -0.127966 \\ \mathrm{C} & 1.966980 & -1.247742 & 1.595247 \\ \mathrm{H} & 2.878753 & -1.354455 & 2.192905 \\ \mathrm{H} & 1.214586 & -0.699557 & 2.167709 \\ \mathrm{H} & 1.568232 & -2.209508 & 1.278524 \\ \mathrm{C} & 0.229495 & 1.485731 & 0.078728 \\ \mathrm{O} & -0.454094 & 2.372793 & -0.681951 \\ \mathrm{O} & 0.228199 & 1.521950 & 1.303307 \\ \mathrm{C} & -1.285344 & 3.292228 & 0.044670 \\ \mathrm{H} & -1.731316 & 3.939998 & -0.711103 \\ \mathrm{H} & -2.061229 & 2.749756 & 0.590717 \\ \mathrm{H} & -0.689616 & 3.877975 & 0.749720\end{array}$

Cis OP1

E(B3LYP 6-31G(d)) Energy=-1073.83064933 Hartrees Number of imaginary frequencies $=0$

$\begin{array}{lccc}\mathrm{O} & 0.913753 & -1.688248 & -0.453498 \\ \mathrm{C} & 0.017978 & -0.789446 & -1.053142 \\ \mathrm{P} & 2.224069 & -0.435368 & -0.000459 \\ \mathrm{C} & 0.797258 & 0.541327 & -0.764205 \\ \mathrm{C} & -1.403621 & -0.874019 & -0.517620 \\ \mathrm{C} & -1.704662 & -1.667937 & 0.591989 \\ \mathrm{C} & -3.013338 & -1.746044 & 1.072777 \\ \mathrm{C} & -4.036441 & -1.030597 & 0.449428 \\ \mathrm{C} & -3.744717 & -0.239611 & -0.664983\end{array}$




$\begin{array}{lccc}\mathrm{C} & -2.437995 & -0.167360 & -1.147218 \\ \mathrm{H} & -0.028729 & -0.930177 & -2.147537 \\ \mathrm{H} & 1.114865 & 1.059347 & -1.675050 \\ \mathrm{H} & -0.902555 & -2.229632 & 1.057919 \\ \mathrm{H} & -3.234077 & -2.370305 & 1.935179 \\ \mathrm{H} & -5.055260 & -1.092870 & 0.823235 \\ \mathrm{H} & -4.536912 & 0.313361 & -1.163909 \\ \mathrm{H} & -2.217806 & 0.444951 & -2.019439 \\ \mathrm{C} & 3.403128 & -1.262491 & -1.152124 \\ \mathrm{H} & 4.441305 & -0.996553 & -0.934976 \\ \mathrm{H} & 3.255919 & -2.342279 & -1.094521 \\ \mathrm{H} & 3.169341 & -0.949327 & -2.175555 \\ \mathrm{C} & 3.314846 & 1.080185 & 0.363167 \\ \mathrm{H} & 2.829869 & 1.736448 & 1.093873 \\ \mathrm{H} & 4.288690 & 0.781502 & 0.769041 \\ \mathrm{H} & 3.494524 & 1.660103 & -0.551229 \\ \mathrm{C} & 2.254169 & -1.110591 & 1.711684 \\ \mathrm{H} & 3.203754 & -0.890390 & 2.207634 \\ \mathrm{H} & 1.444927 & -0.623224 & 2.263066 \\ \mathrm{H} & 2.065920 & -2.184897 & 1.680988 \\ \mathrm{C} & 0.121205 & 1.526805 & 0.155970 \\ \mathrm{O} & -0.513633 & 2.490393 & -0.545437 \\ \mathrm{O} & 0.107184 & 1.466209 & 1.369391 \\ \mathrm{C} & -1.286951 & 3.415954 & 0.239969 \\ \mathrm{H} & -1.699979 & 4.129059 & -0.473964 \\ \mathrm{H} & -2.087103 & 2.887906 & 0.764593 \\ \mathrm{H} & -0.651310 & 3.923617 & 0.969719\end{array}$

Cis TS2

E(B3LYP 6-31G(d)) Energy= -1073.82659739 Hartrees Imaginary Freq $=-49.8740 \mathrm{~cm}^{-1}$

$\begin{array}{lccc}\mathrm{O} & -0.45289 & -1.76435 & -0.81997 \\ \mathrm{C} & 0.27720 & -0.59431 & -1.19586 \\ \mathrm{C} & -0.80002 & 0.44863 & -0.83461 \\ \mathrm{P} & -1.82663 & -1.01752 & 0.01391 \\ \mathrm{C} & 1.65465 & -0.50008 & -0.56284 \\ \mathrm{C} & 2.50931 & 0.55653 & -0.90608 \\ \mathrm{C} & 3.79866 & 0.62878 & -0.38409 \\ \mathrm{C} & 4.25913 & -0.36449 & 0.48438 \\ \mathrm{C} & 3.42101 & -1.42798 & 0.81721\end{array}$




$\begin{array}{lccc}\mathrm{C} & 2.12787 & -1.49742 & 0.29313 \\ \mathrm{H} & 0.41809 & -0.60812 & -2.28690 \\ \mathrm{H} & -1.34709 & 0.76990 & -1.72527 \\ \mathrm{H} & 2.16196 & 1.33117 & -1.58755 \\ \mathrm{H} & 4.44601 & 1.45877 & -0.65584 \\ \mathrm{H} & 5.26557 & -0.31097 & 0.89128 \\ \mathrm{H} & 3.77452 & -2.21199 & 1.48245 \\ \mathrm{H} & 1.48037 & -2.33489 & 0.52929 \\ \mathrm{C} & -0.50817 & 1.64300 & 0.01545 \\ \mathrm{O} & -1.16543 & 2.74281 & -0.44399 \\ \mathrm{O} & 0.17127 & 1.66544 & 1.02377 \\ \mathrm{C} & -1.00900 & 3.92640 & 0.35445 \\ \mathrm{H} & -1.60970 & 4.69259 & -0.13702 \\ \mathrm{H} & -1.36350 & 3.75470 & 1.37464 \\ \mathrm{H} & 0.04112 & 4.22813 & 0.39385 \\ \mathrm{C} & -2.76075 & -2.60269 & -0.32199 \\ \mathrm{H} & -3.62104 & -2.69812 & 0.34774 \\ \mathrm{H} & -3.12703 & -2.59757 & -1.35450 \\ \mathrm{H} & -2.09299 & -3.45899 & -0.20681 \\ \mathrm{C} & -1.33268 & -1.11416 & 1.77921 \\ \mathrm{H} & -0.69374 & -0.26580 & 2.03666 \\ \mathrm{H} & -2.21777 & -1.11489 & 2.42236 \\ \mathrm{H} & -0.76843 & -2.03710 & 1.93395 \\ \mathrm{C} & -3.40321 & 0.02848 & 0.11249 \\ \mathrm{H} & -4.14793 & -0.42378 & 0.77681 \\ \mathrm{H} & -3.17754 & 1.04198 & 0.45921 \\ \mathrm{H} & -3.84907 & 0.11239 & -0.88636\end{array}$

Cis OP2

E(B3LYP 6-31G(d)) Energy=-1073.82909057 Hartrees Number of imaginary frequencies $=0$

$\begin{array}{lccc}\mathrm{O} & -0.516862 & -1.724215 & -0.507644 \\ \mathrm{C} & 0.266505 & -0.635169 & -1.079670 \\ \mathrm{C} & -0.706368 & 0.518567 & -0.853567 \\ \mathrm{P} & -1.929655 & -0.963543 & 0.005881 \\ \mathrm{C} & 1.669781 & -0.589301 & -0.509108 \\ \mathrm{C} & 2.613333 & 0.262440 & -1.098625 \\ \mathrm{C} & 3.924387 & 0.305749 & -0.630301 \\ \mathrm{C} & 4.315929 & -0.513056 & 0.431924 \\ \mathrm{C} & 3.385547 & -1.373121 & 1.013977\end{array}$




$\begin{array}{lccc}\mathrm{C} & 2.070713 & -1.413731 & 0.544655 \\ \mathrm{H} & 0.349168 & -0.838953 & -2.155155 \\ \mathrm{H} & -1.159377 & 0.878197 & -1.778737 \\ \mathrm{H} & 2.317044 & 0.901047 & -1.928762 \\ \mathrm{H} & 4.642004 & 0.976542 & -1.095937 \\ \mathrm{H} & 5.339035 & -0.482915 & 0.797472 \\ \mathrm{H} & 3.682052 & -2.020061 & 1.835912 \\ \mathrm{H} & 1.352487 & -2.094480 & 0.988527 \\ \mathrm{C} & -0.342719 & 1.661427 & 0.008369 \\ \mathrm{O} & -1.074795 & 2.769392 & -0.332818 \\ \mathrm{O} & 0.422193 & 1.662714 & 0.955788 \\ \mathrm{C} & -0.866609 & 3.913443 & 0.504185 \\ \mathrm{H} & -1.519866 & 4.692193 & 0.107047 \\ \mathrm{H} & -1.125445 & 3.691019 & 1.543894 \\ \mathrm{H} & 0.177608 & 4.236707 & 0.468456 \\ \mathrm{C} & -2.554219 & -2.655428 & 0.549147 \\ \mathrm{H} & -3.556390 & -2.573805 & 0.984463 \\ \mathrm{H} & -2.594781 & -3.343796 & -0.300977 \\ \mathrm{H} & -1.882473 & -3.087903 & 1.297502 \\ \mathrm{C} & -2.143314 & -0.100327 & 1.626645 \\ \mathrm{H} & -2.831498 & -0.644993 & 2.278200 \\ \mathrm{H} & -1.168453 & -0.011044 & 2.114331 \\ \mathrm{H} & -2.517221 & 0.912272 & 1.449534 \\ \mathrm{C} & -3.352772 & -0.547459 & -1.104993 \\ \mathrm{H} & -4.275671 & -1.026910 & -0.764707 \\ \mathrm{H} & -3.484225 & 0.536488 & -1.155511 \\ \mathrm{H} & -3.124616 & -0.913790 & -2.112201\end{array}$

Cis TS3

E(B3LYP 6-31G(d)) Energy= -1073.82075445 Hartrees Imaginary Freq $=-204.8307 \mathrm{~cm}^{-1}$

$\begin{array}{lccc}\mathrm{O} & -0.458842 & -1.645856 & -0.766330 \\ \mathrm{C} & 0.298917 & -0.356424 & -1.220899 \\ \mathrm{C} & -0.610588 & 0.787395 & -1.071928 \\ \mathrm{P} & -1.778187 & -1.317975 & 0.085590 \\ \mathrm{C} & 1.663840 & -0.363979 & -0.554021 \\ \mathrm{C} & 2.483870 & 0.765546 & -0.685435 \\ \mathrm{C} & 3.783610 & 0.764196 & -0.185458 \\ \mathrm{C} & 4.288892 & -0.365877 & 0.461951 \\ \mathrm{C} & 3.482278 & -1.494581 & 0.595026\end{array}$




$\begin{array}{lccc}\mathrm{C} & 2.182130 & -1.496782 & 0.082963 \\ \mathrm{H} & 0.429427 & -0.603276 & -2.279728 \\ \mathrm{H} & -1.193896 & 1.041126 & -1.951982 \\ \mathrm{H} & 2.092106 & 1.651343 & -1.175010 \\ \mathrm{H} & 4.400221 & 1.652973 & -0.292392 \\ \mathrm{H} & 5.301279 & -0.364419 & 0.857658 \\ \mathrm{H} & 3.864060 & -2.382819 & 1.092675 \\ \mathrm{H} & 1.568139 & -2.385907 & 0.173811 \\ \mathrm{C} & -0.538270 & 1.801686 & -0.080536 \\ \mathrm{O} & -1.410921 & 2.844162 & -0.379128 \\ \mathrm{O} & 0.106405 & 1.821147 & 0.975694 \\ \mathrm{C} & -1.452496 & 3.886442 & 0.591054 \\ \mathrm{H} & -2.170570 & 4.617390 & 0.211041 \\ \mathrm{H} & -1.777489 & 3.515726 & 1.569695 \\ \mathrm{H} & -0.471266 & 4.355751 & 0.716224 \\ \mathrm{C} & -2.309489 & -3.054657 & 0.358006 \\ \mathrm{H} & -3.191301 & -3.087457 & 1.006510 \\ \mathrm{H} & -2.556001 & -3.524606 & -0.598746 \\ \mathrm{H} & -1.501191 & -3.624362 & 0.823599 \\ \mathrm{C} & -1.500786 & -0.667744 & 1.760842 \\ \mathrm{H} & -2.468299 & -0.427547 & 2.215786 \\ \mathrm{H} & -1.013591 & -1.449924 & 2.351571 \\ \mathrm{H} & -0.863204 & 0.226133 & 1.744592 \\ \mathrm{C} & -3.268431 & -0.581993 & -0.688542 \\ \mathrm{H} & -4.167712 & -1.054805 & -0.272177 \\ \mathrm{H} & -3.287387 & 0.496623 & -0.532853 \\ \mathrm{H} & -3.234505 & -0.777307 & -1.764153\end{array}$

Cis methyl-cinnamate E(B3LYP 6-31G(d)) Energy=-537.524266321 Hartrees Number of imaginary frequencies $=0$

$\begin{array}{lccc}\mathrm{C} & 1.245264 & 0.556323 & -0.000018 \\ \mathrm{C} & 2.504352 & 1.195555 & 0.000074 \\ \mathrm{C} & 3.688798 & 0.466173 & 0.000082 \\ \mathrm{C} & 3.643360 & -0.929393 & -0.000038 \\ \mathrm{C} & 2.406783 & -1.579416 & -0.000150 \\ \mathrm{C} & 1.218195 & -0.853566 & -0.000131 \\ \mathrm{H} & 2.545955 & 2.282465 & 0.000137 \\ \mathrm{H} & 4.644015 & 0.984154 & 0.000182 \\ \mathrm{H} & 4.564763 & -1.505870 & -0.000042\end{array}$




$\begin{array}{lccc}\mathrm{H} & 2.365669 & -2.665484 & -0.000242 \\ \mathrm{H} & 0.263424 & -1.362480 & -0.000137 \\ \mathrm{C} & -1.271135 & 1.276296 & -0.000001 \\ \mathrm{C} & 0.074509 & 1.437534 & -0.000050 \\ \mathrm{H} & 0.368596 & 2.487706 & -0.000141 \\ \mathrm{H} & -1.864146 & 2.185940 & -0.000071 \\ \mathrm{C} & -2.104420 & 0.060589 & 0.000103 \\ \mathrm{O} & -3.415071 & 0.425361 & -0.000171 \\ \mathrm{O} & -1.763076 & -1.109996 & 0.000382 \\ \mathrm{C} & -4.352296 & -0.658685 & -0.000073 \\ \mathrm{H} & -5.338476 & -0.192857 & -0.000233 \\ \mathrm{H} & -4.222472 & -1.282623 & -0.888928 \\ \mathrm{H} & -4.222619 & -1.282339 & 0.889001\end{array}$

Trans Adduct (( $\left.\mathrm{Me}_{3} \mathrm{PCHCOOMe}+\mathrm{PhCHO}\right)$ E(B3LYP 6-31G(d)) Energy=-1073.84336613 Hartrees Number of imaginary frequencies $=0$

$\begin{array}{lccc}\mathrm{C} & -1.18165 & -0.78702 & 1.34087 \\ \mathrm{O} & -0.86869 & -1.94554 & 1.58036 \\ \mathrm{P} & 2.21735 & -1.05533 & -0.40434 \\ \mathrm{C} & 1.19678 & 0.30838 & -0.62228 \\ \mathrm{C} & -2.37280 & -0.39008 & 0.55946 \\ \mathrm{C} & -2.53434 & 0.94961 & 0.17749 \\ \mathrm{C} & -3.65406 & 1.33056 & -0.56115 \\ \mathrm{C} & -4.61611 & 0.37895 & -0.90797 \\ \mathrm{C} & -4.45705 & -0.95932 & -0.52841 \\ \mathrm{C} & -3.33600 & -1.34529 & 0.19871 \\ \mathrm{C} & 2.56577 & -1.47168 & 1.34525 \\ \mathrm{C} & 1.40521 & -2.52157 & -1.13109 \\ \mathrm{C} & 3.87407 & -0.91539 & -1.18669 \\ \mathrm{H} & -0.56713 & 0.05263 & 1.71464 \\ \mathrm{H} & 0.29048 & 0.22643 & -1.20784 \\ \mathrm{H} & -1.76333 & 1.67487 & 0.42727 \\ \mathrm{H} & -3.77589 & 2.36548 & -0.86826 \\ \mathrm{H} & -5.49167 & 0.67807 & -1.47846 \\ \mathrm{H} & -5.20894 & -1.69441 & -0.80262 \\ \mathrm{H} & -3.18611 & -2.37650 & 0.50437 \\ \mathrm{H} & 3.30873 & -2.27228 & 1.42423 \\ \mathrm{H} & 1.63057 & -1.78209 & 1.82091 \\ \mathrm{H} & 2.94421 & -0.56680 & 1.82773\end{array}$




$\begin{array}{lccc}\mathrm{H} & 2.06271 & -3.39359 & -1.06618 \\ \mathrm{H} & 1.16446 & -2.33302 & -2.18134 \\ \mathrm{H} & 0.48348 & -2.72560 & -0.57878 \\ \mathrm{H} & 4.51039 & -1.77554 & -0.95224 \\ \mathrm{H} & 4.33511 & 0.00000 & -0.80544 \\ \mathrm{H} & 3.75760 & -0.82786 & -2.27084 \\ \mathrm{C} & 1.63240 & 1.52911 & -0.04750 \\ \mathrm{O} & 0.72836 & 2.56141 & -0.21029 \\ \mathrm{O} & 2.69519 & 1.70948 & 0.56482 \\ \mathrm{C} & 1.15394 & 3.81776 & 0.31900 \\ \mathrm{H} & 0.34750 & 4.52053 & 0.09711 \\ \mathrm{H} & 1.31831 & 3.76077 & 1.39974 \\ \mathrm{H} & 2.08354 & 4.15194 & -0.15238\end{array}$

Trans TS1

E(B3LYP 6-31G(d)) Energy= -1073.81535677 Hartrees

Imaginary Freq $=-196.3809 \mathrm{~cm}^{-1}$

$\begin{array}{lccc}\mathrm{C} & -0.278837 & -0.834388 & 0.808168 \\ \mathrm{O} & 0.228814 & -2.036586 & 0.769690 \\ \mathrm{P} & 2.289815 & -0.661181 & -0.161558 \\ \mathrm{C} & 0.664947 & 0.135838 & -0.334763 \\ \mathrm{C} & -1.741136 & -0.677418 & 0.398683 \\ \mathrm{C} & -2.511281 & 0.418055 & 0.803067 \\ \mathrm{C} & -3.850065 & 0.526273 & 0.423018 \\ \mathrm{C} & -4.433978 & -0.466121 & -0.367481 \\ \mathrm{C} & -3.673867 & -1.569833 & -0.764242 \\ \mathrm{C} & -2.337882 & -1.675197 & -0.378497 \\ \mathrm{C} & 2.808916 & -0.907504 & 1.566436 \\ \mathrm{C} & 2.377436 & -2.198364 & -1.137394 \\ \mathrm{C} & 3.571219 & 0.429952 & -0.898293 \\ \mathrm{H} & -0.106853 & -0.242792 & 1.740670 \\ \mathrm{H} & 0.244389 & -0.100221 & -1.313366 \\ \mathrm{H} & -2.064312 & 1.184432 & 1.434229 \\ \mathrm{H} & -4.439913 & 1.379742 & 0.749517 \\ \mathrm{H} & -5.477686 & -0.386024 & -0.661550 \\ \mathrm{H} & -4.128918 & -2.353961 & -1.365342 \\ \mathrm{H} & -1.733016 & -2.537527 & -0.642800 \\ \mathrm{H} & 3.852398 & -1.237589 & 1.593443 \\ \mathrm{H} & 2.138593 & -1.652381 & 1.997423 \\ \mathrm{H} & 2.712873 & 0.049612 & 2.086032\end{array}$




$\begin{array}{lccc}\mathrm{H} & 3.332317 & -2.701268 & -0.951762 \\ \mathrm{H} & 2.304622 & -1.955303 & -2.202685 \\ \mathrm{H} & 1.536668 & -2.817649 & -0.822358 \\ \mathrm{H} & 4.555862 & -0.042953 & -0.819317 \\ \mathrm{H} & 3.581212 & 1.384877 & -0.368402 \\ \mathrm{H} & 3.350933 & 0.609345 & -1.955073 \\ \mathrm{C} & 0.707458 & 1.584630 & -0.044699 \\ \mathrm{O} & -0.236453 & 2.274258 & -0.722544 \\ \mathrm{O} & 1.448267 & 2.106357 & 0.782449 \\ \mathrm{C} & -0.358687 & 3.661954 & -0.376256 \\ \mathrm{H} & -1.169861 & 4.045603 & -0.995621 \\ \mathrm{H} & -0.599011 & 3.775996 & 0.684185 \\ \mathrm{H} & 0.571991 & 4.196291 & -0.587098\end{array}$

Trans OP1

E(B3LYP 6-31G(d)) Energy=-1073.83422892 Hartrees Number of imaginary frequencies $=0$

$\begin{array}{lccc}\mathrm{C} & -0.512145 & -0.270306 & 0.773821 \\ \mathrm{O} & -0.079515 & -1.620498 & 0.768317 \\ \mathrm{P} & 1.535938 & -1.334158 & -0.034761 \\ \mathrm{C} & 0.525161 & 0.291059 & -0.219774 \\ \mathrm{C} & -1.960782 & -0.093600 & 0.362267 \\ \mathrm{C} & -2.622372 & 1.111309 & 0.633486 \\ \mathrm{C} & -3.942439 & 1.304950 & 0.227414 \\ \mathrm{C} & -4.622615 & 0.289230 & -0.448861 \\ \mathrm{C} & -3.973205 & -0.918057 & -0.711632 \\ \mathrm{C} & -2.649938 & -1.108330 & -0.308941 \\ \mathrm{C} & 2.475267 & -1.491016 & 1.537400 \\ \mathrm{C} & 1.317947 & -2.950080 & -0.912819 \\ \mathrm{C} & 2.986171 & -0.760166 & -1.128284 \\ \mathrm{H} & -0.360124 & 0.200397 & 1.757216 \\ \mathrm{H} & 0.140825 & 0.349233 & -1.242654 \\ \mathrm{H} & -2.100122 & 1.900642 & 1.171318 \\ \mathrm{H} & -4.442929 & 2.245055 & 0.445643 \\ \mathrm{H} & -5.653540 & 0.435800 & -0.760580 \\ \mathrm{H} & -4.500147 & -1.717579 & -1.226874 \\ \mathrm{H} & -2.141934 & -2.049721 & -0.490657 \\ \mathrm{H} & 3.523371 & -1.746175 & 1.358186 \\ \mathrm{H} & 1.995817 & -2.241334 & 2.168655 \\ \mathrm{H} & 2.431033 & -0.525155 & 2.052237\end{array}$




$\begin{array}{lccc}\mathrm{H} & 2.279002 & -3.367120 & -1.226298 \\ \mathrm{H} & 0.705527 & -2.781119 & -1.805516 \\ \mathrm{H} & 0.782308 & -3.643846 & -0.262669 \\ \mathrm{H} & 3.776441 & -1.518844 & -1.169535 \\ \mathrm{H} & 3.426827 & 0.167870 & -0.746922 \\ \mathrm{H} & 2.645793 & -0.567280 & -2.153161 \\ \mathrm{C} & 1.235842 & 1.559715 & 0.148158 \\ \mathrm{O} & 1.536635 & 2.304526 & -0.940118 \\ \mathrm{O} & 1.541364 & 1.883599 & 1.280731 \\ \mathrm{C} & 2.265196 & 3.515710 & -0.676614 \\ \mathrm{H} & 2.420140 & 3.980800 & -1.650465 \\ \mathrm{H} & 1.688882 & 4.174831 & -0.022231 \\ \mathrm{H} & 3.223352 & 3.292396 & -0.199447\end{array}$

Trans TS2

E(B3LYP 6-31G(d)) Energy= -1073.83083694 Hartrees Imaginary Freq $=-52.4418 \mathrm{~cm}^{-1}$

$\begin{array}{lccc}\mathrm{O} & 0.142205 & -1.292858 & -1.063459 \\ \mathrm{C} & 0.482282 & 0.079563 & -0.859757 \\ \mathrm{C} & -0.606094 & 0.444151 & 0.154083 \\ \mathrm{P} & -1.134887 & -1.455609 & 0.161944 \\ \mathrm{C} & 1.911495 & 0.283003 & -0.385280 \\ \mathrm{C} & 2.330104 & 1.530826 & 0.097636 \\ \mathrm{C} & 3.650152 & 1.733185 & 0.499562 \\ \mathrm{C} & 4.574410 & 0.688539 & 0.421602 \\ \mathrm{C} & 4.167638 & -0.554169 & -0.065189 \\ \mathrm{C} & 2.845458 & -0.754848 & -0.468283 \\ \mathrm{H} & 0.331215 & 0.625794 & -1.800665 \\ \mathrm{H} & -0.258704 & 0.752472 & 1.143591 \\ \mathrm{H} & 1.618484 & 2.351965 & 0.159296 \\ \mathrm{H} & 3.957090 & 2.706243 & 0.874836 \\ \mathrm{H} & 5.603161 & 0.844535 & 0.735621 \\ \mathrm{H} & 4.881643 & -1.371202 & -0.134862 \\ \mathrm{H} & 2.522500 & -1.715426 & -0.856175 \\ \mathrm{C} & -1.667081 & 1.375464 & -0.344420 \\ \mathrm{O} & -2.206368 & 2.102681 & 0.666481 \\ \mathrm{O} & -2.045124 & 1.462974 & -1.496782 \\ \mathrm{C} & -3.286136 & 2.971490 & 0.288365 \\ \mathrm{H} & -2.953513 & 3.695131 & -0.460348 \\ \mathrm{H} & -4.118764 & 2.394685 & -0.124163\end{array}$




$\begin{array}{lrrr}\mathrm{H} & -3.588785 & 3.478582 & 1.205146 \\ \mathrm{C} & -1.845755 & -2.812020 & -0.902231 \\ \mathrm{H} & -2.526210 & -3.449020 & -0.329395 \\ \mathrm{H} & -2.406233 & -2.352730 & -1.723541 \\ \mathrm{H} & -1.038163 & -3.403553 & -1.338270 \\ \mathrm{C} & -0.222505 & -2.262089 & 1.542018 \\ \mathrm{H} & -0.813283 & -3.093571 & 1.939407 \\ \mathrm{H} & 0.739793 & -2.633508 & 1.184741 \\ \mathrm{H} & -0.047421 & -1.544560 & 2.349345 \\ \mathrm{C} & -2.776897 & -1.131719 & 1.057043 \\ \mathrm{H} & -3.197189 & -2.058562 & 1.463588 \\ \mathrm{H} & -2.643802 & -0.409571 & 1.869073 \\ \mathrm{H} & -3.506655 & -0.714179 & 0.352797\end{array}$

Trans OP2

E(B3LYP 6-31G(d)) Energy=-1073.83666266 Hartrees Number of imaginary frequencies $=0$

$\begin{array}{lccc}\mathrm{O} & 0.108996 & -1.431696 & -0.848148 \\ \mathrm{C} & 0.501195 & -0.028274 & -0.759373 \\ \mathrm{C} & -0.539094 & 0.458430 & 0.228189 \\ \mathrm{P} & -1.313583 & -1.484327 & 0.054121 \\ \mathrm{C} & 1.951570 & 0.122910 & -0.351715 \\ \mathrm{C} & 2.502663 & 1.410620 & -0.276735 \\ \mathrm{C} & 3.830615 & 1.594201 & 0.103090 \\ \mathrm{C} & 4.631227 & 0.490844 & 0.411603 \\ \mathrm{C} & 4.092216 & -0.792567 & 0.331039 \\ \mathrm{C} & 2.760377 & -0.976853 & -0.050761 \\ \mathrm{H} & 0.339137 & 0.429102 & -1.741733 \\ \mathrm{H} & 1.884581 & 2.272551 & -0.519220 \\ \mathrm{H} & 4.242219 & 2.598875 & 0.157109 \\ \mathrm{H} & 5.667355 & 0.632999 & 0.707313 \\ \mathrm{H} & 4.709270 & -1.657562 & 0.561466 \\ \mathrm{H} & 2.345008 & -1.975924 & -0.128219 \\ \mathrm{H} & -0.161843 & 0.608877 & 1.241288 \\ \mathrm{C} & -1.411887 & -3.323848 & -0.335502 \\ \mathrm{H} & -2.328409 & -3.757925 & 0.079511 \\ \mathrm{H} & -1.402380 & -3.487370 & -1.417429 \\ \mathrm{H} & -0.552546 & -3.852575 & 0.090101 \\ \mathrm{C} & -1.487286 & -1.652311 & 1.893746 \\ \mathrm{H} & -1.916804 & -0.736995 & 2.309325\end{array}$




$\begin{array}{lrrr}\mathrm{H} & -2.099488 & -2.519293 & 2.160757 \\ \mathrm{H} & -0.488235 & -1.787642 & 2.323174 \\ \mathrm{C} & -2.929744 & -0.971875 & -0.675873 \\ \mathrm{H} & -3.406643 & -0.235078 & -0.023750 \\ \mathrm{H} & -2.744619 & -0.493149 & -1.642188 \\ \mathrm{H} & -3.592855 & -1.829132 & -0.818296 \\ \mathrm{C} & -1.393733 & 1.582647 & -0.197652 \\ \mathrm{O} & -1.547132 & 1.989515 & -1.337941 \\ \mathrm{O} & -2.089202 & 2.102160 & 0.858394 \\ \mathrm{C} & -2.992734 & 3.165699 & 0.528785 \\ \mathrm{H} & -3.457119 & 3.460025 & 1.471241 \\ \mathrm{H} & -2.454471 & 4.009916 & 0.088837 \\ \mathrm{H} & -3.752019 & 2.827632 & -0.182594\end{array}$

Trans TS3

E(B3LYP 6-31G(d)) Energy= -1073.82813922 Hartrees Imaginary Freq $=-182.0550 \mathrm{~cm}^{-1}$

$\begin{array}{lccc}\mathrm{O} & -0.162276 & 1.443189 & -0.462923 \\ \mathrm{C} & -0.465229 & -0.097844 & -0.543744 \\ \mathrm{C} & 0.477137 & -0.811295 & 0.315250 \\ \mathrm{P} & 1.288685 & 1.747495 & 0.135751 \\ \mathrm{C} & -1.947341 & -0.218835 & -0.240973 \\ \mathrm{C} & -2.449362 & -1.440050 & 0.228173 \\ \mathrm{C} & -3.815653 & -1.611072 & 0.456726 \\ \mathrm{C} & -4.703854 & -0.560888 & 0.223038 \\ \mathrm{C} & -4.214328 & 0.658841 & -0.247553 \\ \mathrm{C} & -2.849413 & 0.827196 & -0.482693 \\ \mathrm{H} & -0.260346 & -0.308039 & -1.597671 \\ \mathrm{H} & -1.759082 & -2.257900 & 0.411004 \\ \mathrm{H} & -4.183611 & -2.566659 & 0.821717 \\ \mathrm{H} & -5.767582 & -0.691174 & 0.404721 \\ \mathrm{H} & -4.897174 & 1.484080 & -0.434416 \\ \mathrm{H} & -2.477157 & 1.778623 & -0.846344 \\ \mathrm{H} & 0.182132 & -1.025693 & 1.338121 \\ \mathrm{C} & 1.214461 & 3.578282 & 0.039611 \\ \mathrm{H} & 2.173623 & 4.015894 & 0.336168 \\ \mathrm{H} & 0.978729 & 3.888526 & -0.981624 \\ \mathrm{H} & 0.432125 & 3.955023 & 0.705133 \\ \mathrm{C} & 1.662835 & 1.466599 & 1.907588 \\ \mathrm{H} & 2.180363 & 0.516878 & 2.040264\end{array}$




$\begin{array}{lccc}\mathrm{H} & 2.265349 & 2.299542 & 2.293274 \\ \mathrm{H} & 0.719370 & 1.426627 & 2.459063 \\ \mathrm{C} & 2.690096 & 1.291524 & -0.930903 \\ \mathrm{H} & 3.621969 & 1.385942 & -0.362610 \\ \mathrm{H} & 2.587177 & 0.264464 & -1.305673 \\ \mathrm{H} & 2.718238 & 1.986242 & -1.776600 \\ \mathrm{C} & 1.481662 & -1.635648 & -0.258087 \\ \mathrm{O} & 1.867772 & -1.648457 & -1.437196 \\ \mathrm{O} & 2.101520 & -2.433825 & 0.688259 \\ \mathrm{C} & 3.154904 & -3.253563 & 0.188701 \\ \mathrm{H} & 3.521025 & -3.821912 & 1.047197 \\ \mathrm{H} & 2.795964 & -3.936681 & -0.587762 \\ \mathrm{H} & 3.966991 & -2.652965 & -0.236165\end{array}$

Trans metil-cinnamate E(B3LYP 6-31G(d)) Energy=-537.531250284 Hartrees Number of imaginary frequencies $=0$

$\begin{array}{lccc}\mathrm{C} & -1.378143 & 0.189826 & 0.000028 \\ \mathrm{C} & -2.313657 & 1.240485 & 0.000445 \\ \mathrm{C} & -3.683441 & 0.984526 & 0.000428 \\ \mathrm{C} & -4.146399 & -0.331715 & -0.000051 \\ \mathrm{C} & -3.229829 & -1.388644 & -0.000467 \\ \mathrm{C} & -1.863024 & -1.132605 & -0.000431 \\ \mathrm{H} & -1.954430 & 2.266790 & 0.000813 \\ \mathrm{H} & -4.387916 & 1.811758 & 0.000780 \\ \mathrm{H} & -5.213623 & -0.535837 & -0.000114 \\ \mathrm{H} & -3.585035 & -2.415626 & -0.000835 \\ \mathrm{H} & -1.164647 & -1.964235 & -0.000845 \\ \mathrm{C} & 1.091930 & -0.326986 & 0.000452 \\ \mathrm{C} & 0.046956 & 0.520982 & -0.000083 \\ \mathrm{H} & 0.288871 & 1.583145 & -0.000686 \\ \mathrm{H} & 0.982678 & -1.406699 & 0.001258 \\ \mathrm{C} & 2.471289 & 0.198010 & -0.000001 \\ \mathrm{O} & 3.370235 & -0.819509 & 0.000749 \\ \mathrm{O} & 2.793501 & 1.371701 & -0.001008 \\ \mathrm{C} & 4.746314 & -0.416096 & 0.000033 \\ \mathrm{H} & 5.323906 & -1.341300 & 0.000804 \\ \mathrm{H} & 4.973941 & 0.178008 & -0.889537 \\ \mathrm{H} & 4.974378 & 0.179767 & 0.888316\end{array}$




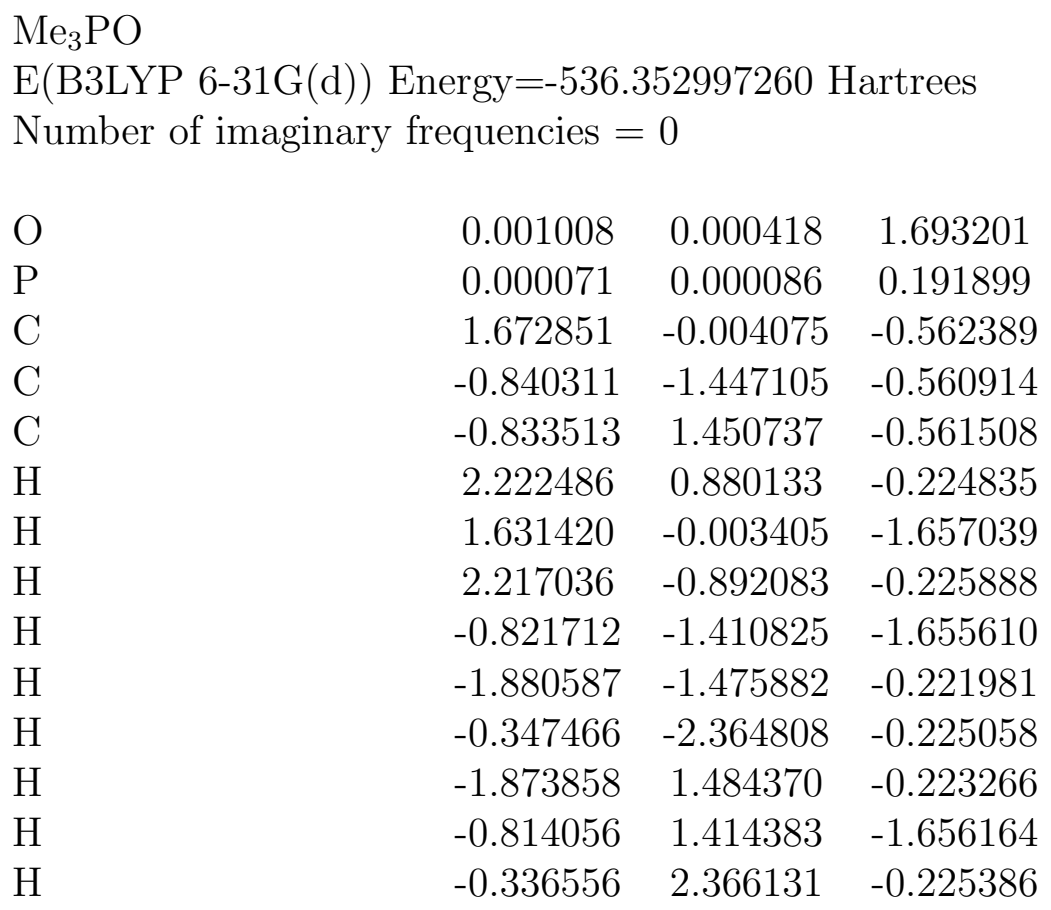

\section{Semi-Stabilized Ylides $\left(\mathrm{Me}_{3} \mathrm{PCHPh}+\mathrm{PhCHO}\right)$}

\begin{tabular}{lccc}
\hline $\mathrm{Me}_{3} \mathrm{PCHPh}$ \\
$\mathrm{E}$ (B3LYP 6-31G(d)) Energy=-731.415168909 Hartree \\
\multicolumn{4}{c}{ Number of imaginary frequencies $=0$} \\
$\mathrm{C}$ & 0.380455 & 0.967333 & -0.001488 \\
$\mathrm{P}$ & 1.818400 & 0.071517 & 0.000012 \\
$\mathrm{C}$ & 2.122781 & -1.055232 & -1.438207 \\
$\mathrm{C}$ & 3.237956 & 1.226647 & 0.000107 \\
$\mathrm{C}$ & 2.120958 & -1.053675 & 1.439875 \\
$\mathrm{H}$ & 0.528368 & 2.043297 & -0.001351 \\
$\mathrm{H}$ & 2.187148 & -0.452710 & -2.348944 \\
$\mathrm{H}$ & 1.277812 & -1.741428 & -1.546024 \\
$\mathrm{H}$ & 3.042308 & -1.638546 & -1.316041 \\
$\mathrm{H}$ & 4.180209 & 0.671049 & 0.000970 \\
$\mathrm{H}$ & 3.196916 & 1.861496 & 0.889238 \\
$\mathrm{H}$ & 3.197897 & 1.860437 & -0.889822 \\
$\mathrm{H}$ & 3.039973 & -1.638066 & 1.319101 \\
$\mathrm{H}$ & 1.275178 & -1.738825 & 1.548012
\end{tabular}




$\begin{array}{lccc}\mathrm{H} & 2.185290 & -0.450004 & 2.349859 \\ \mathrm{C} & -3.693010 & -0.430580 & 0.000450 \\ \mathrm{C} & -2.639849 & -1.347268 & -0.000152 \\ \mathrm{C} & -1.315316 & -0.917061 & -0.000789 \\ \mathrm{C} & -0.974242 & 0.459181 & -0.000791 \\ \mathrm{C} & -2.063485 & 1.367321 & -0.000219 \\ \mathrm{C} & -3.384071 & 0.933422 & 0.000364 \\ \mathrm{H} & -4.725343 & -0.768736 & 0.000932 \\ \mathrm{H} & -2.849298 & -2.415252 & -0.000134 \\ \mathrm{H} & -0.530234 & -1.671604 & -0.001393 \\ \mathrm{H} & -1.851034 & 2.434632 & -0.000258 \\ \mathrm{H} & -4.184247 & 1.670979 & 0.000772\end{array}$

\section{$\mathrm{PhCHO}$}

E(B3LYP 6-31G(d)) Energy=-345.573455692 Hartrees Number of imaginary frequencies $=0$

$\begin{array}{lccc}\mathrm{C} & 0.592145 & -2.150954 & 0.000000 \\ \mathrm{C} & 1.630339 & -1.216571 & 0.000000 \\ \mathrm{C} & 1.333323 & 0.145608 & 0.000000 \\ \mathrm{C} & 0.000000 & 0.575208 & 0.000000 \\ \mathrm{C} & -1.039168 & -0.368003 & 0.000000 \\ \mathrm{C} & -0.742102 & -1.726587 & 0.000000 \\ \mathrm{H} & 0.821246 & -3.213358 & 0.000000 \\ \mathrm{H} & 2.664247 & -1.550104 & 0.000000 \\ \mathrm{H} & 2.133766 & 0.882781 & 0.000000 \\ \mathrm{H} & -2.064294 & -0.010180 & 0.000000 \\ \mathrm{H} & -1.544252 & -2.459452 & 0.000000 \\ \mathrm{C} & -0.305965 & 2.023605 & 0.000000 \\ \mathrm{O} & -1.426439 & 2.496397 & 0.000000 \\ \mathrm{H} & 0.589368 & 2.685305 & 0.000000\end{array}$

Cis Adduct $\left(\mathrm{Me}_{3} \mathrm{PCHPh}+\mathrm{PhCHO}\right)$ $\mathrm{E}(\mathrm{B} 3 \mathrm{LYP}$ 6-31G(d)) Energy=-1076.99853732 Hartrees Number of imaginary frequencies $=0$

$\begin{array}{llll}\mathrm{O} & -1.20111 & -2.37991 & 0.26693 \\ \mathrm{C} & -1.47122 & -1.19818 & 0.09193 \\ \mathrm{P} & 2.56277 & -1.43661 & -0.14137 \\ \mathrm{C} & 1.80468 & -0.12326 & -0.91299\end{array}$




$\begin{array}{lccc}\mathrm{C} & -2.84266 & -0.65056 & 0.06997 \\ \mathrm{C} & 1.69251 & 1.24036 & -0.45038 \\ \mathrm{C} & -3.95548 & -1.48839 & 0.24497 \\ \mathrm{C} & -5.23748 & -0.95072 & 0.22232 \\ \mathrm{C} & -5.41477 & 0.42467 & 0.02592 \\ \mathrm{C} & -4.31083 & 1.26261 & -0.14798 \\ \mathrm{C} & -3.02396 & 0.72614 & -0.12639 \\ \mathrm{C} & 2.33358 & 1.75458 & 0.70642 \\ \mathrm{C} & 2.15849 & 3.07226 & 1.12084 \\ \mathrm{C} & 1.34750 & 3.95296 & 0.40126 \\ \mathrm{C} & 0.71700 & 3.47814 & -0.75325 \\ \mathrm{C} & 0.88143 & 2.16036 & -1.16789 \\ \mathrm{H} & -0.66021 & -0.46211 & -0.06698 \\ \mathrm{H} & 1.36651 & -0.37571 & -1.87473 \\ \mathrm{H} & -3.78748 & -2.55055 & 0.39592 \\ \mathrm{H} & -6.10198 & -1.59515 & 0.35701 \\ \mathrm{H} & -6.41815 & 0.84234 & 0.00921 \\ \mathrm{H} & -4.45399 & 2.32890 & -0.29869 \\ \mathrm{H} & -2.15423 & 1.36684 & -0.25939 \\ \mathrm{H} & 2.99738 & 1.11557 & 1.28616 \\ \mathrm{H} & 2.67265 & 3.41727 & 2.01581 \\ \mathrm{H} & 1.21782 & 4.98162 & 0.72508 \\ \mathrm{H} & 0.08983 & 4.14465 & -1.34242 \\ \mathrm{H} & 0.38402 & 1.81831 & -2.07382 \\ \mathrm{C} & 2.15711 & -2.96149 & -1.06328 \\ \mathrm{H} & 2.71973 & -3.81030 & -0.66346 \\ \mathrm{H} & 1.08555 & -3.15337 & -0.95906 \\ \mathrm{H} & 2.40664 & -2.83529 & -2.12058 \\ \mathrm{C} & 4.41289 & -1.41913 & -0.01457 \\ \mathrm{H} & 4.79528 & -2.29836 & 0.51650 \\ \mathrm{H} & 4.83622 & -1.38767 & -1.02299 \\ \mathrm{H} & 4.73309 & -0.51685 & 0.51554 \\ \mathrm{H} & 2.03362 & -1.77082 & 1.59253 \\ \mathrm{H} & 2.65930 & -2.53803 & 2.06106 \\ & 2.09161 & -0.85322 & 2.18418 \\ \mathrm{H} & -2.10762 & 1.56419\end{array}$

Cis TS1

E(B3LYP 6-31G(d)) Energy= -1076.97922207 Hartrees Imaginary Freq $=-195.3779 \mathrm{~cm}^{-1}$ 


\begin{tabular}{|c|c|c|c|}
\hline $\mathrm{O}$ & 0.358171 & -2.054608 & 1.262645 \\
\hline $\mathrm{C}$ & -0.102611 & -1.777496 & 0.108027 \\
\hline $\mathrm{P}$ & 2.522612 & -0.522712 & -0.160204 \\
\hline $\mathrm{C}$ & 0.849444 & -0.193828 & -0.672623 \\
\hline $\mathrm{C}$ & -1.556877 & -1.383923 & -0.047583 \\
\hline $\mathrm{C}$ & 0.315977 & 1.142107 & -0.280578 \\
\hline $\mathrm{C}$ & -2.334367 & -1.161381 & 1.092889 \\
\hline $\mathrm{C}$ & -3.688389 & -0.844413 & 0.976603 \\
\hline $\mathrm{C}$ & -4.281545 & -0.750356 & -0.283984 \\
\hline $\mathrm{C}$ & -3.514020 & -0.983716 & -1.428943 \\
\hline $\mathrm{C}$ & -2.162217 & -1.301509 & -1.307383 \\
\hline $\mathrm{C}$ & 0.014646 & 1.446468 & 1.062614 \\
\hline $\mathrm{C}$ & -0.511222 & 2.688229 & 1.416739 \\
\hline $\mathrm{C}$ & -0.766362 & 3.655571 & 0.442582 \\
\hline $\mathrm{C}$ & -0.500278 & 3.360758 & -0.896074 \\
\hline $\mathrm{C}$ & 0.035677 & 2.123571 & -1.250560 \\
\hline $\mathrm{H}$ & 0.202371 & -2.419373 & -0.753207 \\
\hline $\mathrm{H}$ & 0.811607 & -0.341560 & -1.758597 \\
\hline $\mathrm{H}$ & -1.855261 & -1.269415 & 2.061048 \\
\hline $\mathrm{H}$ & -4.284652 & -0.676760 & 1.870661 \\
\hline $\mathrm{H}$ & -5.336991 & -0.505882 & -0.375687 \\
\hline $\mathrm{H}$ & -3.972380 & -0.923685 & -2.413377 \\
\hline $\mathrm{H}$ & -1.568676 & -1.495359 & -2.200013 \\
\hline $\mathrm{H}$ & 0.152542 & 0.679860 & 1.817953 \\
\hline $\mathrm{H}$ & -0.740338 & 2.893638 & 2.459665 \\
\hline $\mathrm{H}$ & -1.179821 & 4.621197 & 0.720990 \\
\hline $\mathrm{H}$ & -0.707502 & 4.097610 & -1.668453 \\
\hline $\mathrm{H}$ & 0.239101 & 1.907455 & -2.297539 \\
\hline $\mathrm{C}$ & 2.960206 & -2.258589 & -0.510895 \\
\hline $\mathrm{H}$ & 4.016902 & -2.435199 & -0.288380 \\
\hline $\mathrm{H}$ & 2.325268 & -2.868236 & 0.139501 \\
\hline $\mathrm{H}$ & 2.766870 & -2.500378 & -1.559719 \\
\hline $\mathrm{C}$ & 3.777859 & 0.517672 & -1.010990 \\
\hline $\mathrm{H}$ & 4.783334 & 0.314602 & -0.625730 \\
\hline $\mathrm{H}$ & 3.760223 & 0.317675 & -2.086838 \\
\hline $\mathrm{H}$ & 3.536697 & 1.572666 & -0.849858 \\
\hline $\mathrm{C}$ & 2.778594 & -0.275383 & 1.624100 \\
\hline $\mathrm{H}$ & 3.792009 & -0.587915 & 1.895210 \\
\hline $\mathrm{H}$ & 2.636226 & 0.774801 & 1.888820 \\
\hline $\mathrm{H}$ & 2.030833 & -0.900888 & 2.121804 \\
\hline
\end{tabular}




\section{Cis OP1}

E(B3LYP 6-31G(d)) Energy=-1077.00611365 Hartrees

Number of imaginary frequencies $=0$

$\begin{array}{lccc}\mathrm{O} & 0.620328 & -1.956879 & 0.281283 \\ \mathrm{C} & -0.116445 & -1.310304 & -0.733126 \\ \mathrm{P} & 2.163127 & -0.935879 & 0.062540 \\ \mathrm{C} & 0.784957 & -0.051129 & -0.918210 \\ \mathrm{C} & -1.586885 & -1.129272 & -0.402299 \\ \mathrm{C} & 0.366215 & 1.286627 & -0.361132 \\ \mathrm{C} & -2.128174 & -1.690979 & 0.758063 \\ \mathrm{C} & -3.491426 & -1.566986 & 1.037340 \\ \mathrm{C} & -4.330315 & -0.881694 & 0.158157 \\ \mathrm{C} & -3.797763 & -0.323360 & -1.006989 \\ \mathrm{C} & -2.437886 & -0.451253 & -1.285556 \\ \mathrm{C} & -0.209638 & 1.418698 & 0.914590 \\ \mathrm{C} & -0.560113 & 2.671964 & 1.414410 \\ \mathrm{C} & -0.340610 & 3.823038 & 0.654686 \\ \mathrm{C} & 0.228399 & 3.708170 & -0.613763 \\ \mathrm{C} & 0.575623 & 2.452193 & -1.113634 \\ \mathrm{H} & -0.074544 & -1.884489 & -1.679439 \\ \mathrm{H} & 1.117928 & 0.089707 & -1.953985 \\ \mathrm{H} & -1.465261 & -2.227056 & 1.428999 \\ \mathrm{H} & -3.898297 & -2.010565 & 1.943021 \\ \mathrm{H} & -5.390957 & -0.784322 & 0.375291 \\ \mathrm{H} & -4.442912 & 0.212354 & -1.698958 \\ \mathrm{H} & -2.032668 & -0.014224 & -2.196161 \\ \mathrm{H} & -0.403311 & 0.531140 & 1.507661 \\ \mathrm{H} & -1.010600 & 2.748016 & 2.400631 \\ \mathrm{H} & -0.615127 & 4.798834 & 1.046294 \\ \mathrm{H} & 0.399728 & 4.594847 & -1.218755 \\ \mathrm{H} & 1.014296 & 2.373141 & -2.106043 \\ \mathrm{C} & 3.009224 & -2.358400 & -0.754976 \\ \mathrm{H} & 4.094034 & -2.226860 & -0.793144 \\ \mathrm{H} & 2.747429 & -3.276666 & -0.226159 \\ \mathrm{H} & 2.632330 & -2.447741 & -1.779728 \\ \mathrm{C} & 3.519489 & 0.353156 & -0.291965 \\ \mathrm{H} & 4.482277 & 0.052458 & 0.138041 \\ \mathrm{H} & 0.477602 & -1.373405 \\ & & & 0.122367 \\ \mathrm{H} & -0.932614 & 1.893703 \\ \mathrm{H} & -0.839155 & 2.154723\end{array}$




$\begin{array}{llll}\mathrm{H} & 1.859820 & -0.069825 & 2.308154 \\ \mathrm{H} & 1.961803 & -1.841895 & 2.316433\end{array}$

Cis TS2

E(B3LYP 6-31G(d)) Energy $=-1077.00150825$ Hartrees Imaginary Freq $=-20.1310 \mathrm{~cm}^{-1}$

$\begin{array}{lccc}\mathrm{O} & 0.95454 & -1.91143 & -0.20575 \\ \mathrm{C} & 0.03738 & -1.12205 & -0.99430 \\ \mathrm{P} & 2.22593 & -0.78588 & 0.07634 \\ \mathrm{C} & 0.77347 & 0.23314 & -0.92922 \\ \mathrm{C} & -1.39208 & -1.25862 & -0.51141 \\ \mathrm{C} & 0.14622 & 1.46306 & -0.35035 \\ \mathrm{C} & -1.72226 & -2.07542 & 0.57500 \\ \mathrm{C} & -3.05353 & -2.21991 & 0.97459 \\ \mathrm{C} & -4.07060 & -1.55554 & 0.28940 \\ \mathrm{C} & -3.74900 & -0.74487 & -0.80249 \\ \mathrm{C} & -2.42119 & -0.60039 & -1.19968 \\ \mathrm{C} & -0.58520 & 1.46788 & 0.85291 \\ \mathrm{C} & -1.10311 & 2.65061 & 1.37826 \\ \mathrm{C} & -0.90056 & 3.86738 & 0.72333 \\ \mathrm{C} & -0.17649 & 3.88550 & -0.46913 \\ \mathrm{C} & 0.33353 & 2.69889 & -0.99603 \\ \mathrm{H} & 0.07198 & -1.50261 & -2.02548 \\ \mathrm{H} & 1.16984 & 0.48903 & -1.91807 \\ \mathrm{H} & -0.92935 & -2.60150 & 1.09569 \\ \mathrm{H} & -3.29430 & -2.85920 & 1.82046 \\ \mathrm{H} & -5.10609 & -1.66953 & 0.59938 \\ \mathrm{H} & -4.53385 & -0.22348 & -1.34454 \\ \mathrm{H} & -2.17823 & 0.03684 & -2.04697 \\ \mathrm{H} & -0.76061 & 0.53530 & 1.37941 \\ \mathrm{H} & -1.66909 & 2.61995 & 2.30599 \\ \mathrm{H} & -1.30486 & 4.78791 & 1.13584 \\ \mathrm{H} & -0.01369 & 4.82254 & -0.99605 \\ \mathrm{H} & 0.88741 & 2.72652 & -1.93257 \\ \mathrm{C} & 3.05450 & -2.17739 & 1.05700 \\ \mathrm{H} & 4.06699 & -1.89117 & 1.36145 \\ \mathrm{H} & 2.47339 & -2.40361 & 1.95757 \\ \mathrm{H} & 3.10681 & -3.09049 & 0.45698 \\ \mathrm{C} & 3.52526 & -0.59872 & -1.23131 \\ \mathrm{H} & 4.51199 & -0.87968 & -0.85181\end{array}$




$\begin{array}{lccc}\mathrm{H} & 3.27777 & -1.25931 & -2.06971 \\ \mathrm{H} & 3.55217 & 0.43054 & -1.59963 \\ \mathrm{C} & 2.58712 & 0.52729 & 1.35301 \\ \mathrm{H} & 3.56318 & 0.34684 & 1.81298 \\ \mathrm{H} & 2.55667 & 1.52224 & 0.90306 \\ \mathrm{H} & 1.82574 & 0.49754 & 2.13950\end{array}$

Cis OP2

E(B3LYP 6-31G(d)) Energy=-1077.00585468 Hartrees Number of imaginary frequencies $=0$

$\begin{array}{lccc}\mathrm{O} & -0.80983 & -2.10730 & -0.48012 \\ \mathrm{C} & 0.02706 & -1.16578 & -1.10871 \\ \mathrm{P} & -2.10752 & -0.91407 & 0.11242 \\ \mathrm{C} & -0.85374 & 0.11213 & -0.89534 \\ \mathrm{C} & 1.45155 & -1.15301 & -0.57588 \\ \mathrm{C} & -0.33382 & 1.41684 & -0.35407 \\ \mathrm{C} & 1.82725 & -1.98883 & 0.48012 \\ \mathrm{C} & 3.14528 & -2.00078 & 0.94442 \\ \mathrm{C} & 4.10644 & -1.18005 & 0.35385 \\ \mathrm{C} & 3.74263 & -0.35102 & -0.71088 \\ \mathrm{C} & 2.42762 & -0.34259 & -1.17293 \\ \mathrm{C} & 0.38338 & 1.50172 & 0.85197 \\ \mathrm{C} & 0.82440 & 2.73141 & 1.33882 \\ \mathrm{C} & 0.56130 & 3.90657 & 0.63186 \\ \mathrm{C} & -0.14492 & 3.84051 & -0.56942 \\ \mathrm{C} & -0.58560 & 2.60817 & -1.05275 \\ \mathrm{H} & 0.09466 & -1.36748 & -2.19233 \\ \mathrm{H} & -1.38922 & 0.32911 & -1.82981 \\ \mathrm{H} & 1.07632 & -2.64009 & 0.91443 \\ \mathrm{H} & 3.42208 & -2.65961 & 1.76418 \\ \mathrm{H} & 5.13236 & -1.19009 & 0.71305 \\ \mathrm{H} & 4.48513 & 0.28775 & -1.18278 \\ \mathrm{H} & 2.15476 & 0.30439 & -2.00420 \\ \mathrm{H} & 0.61710 & 0.59695 & 1.40353 \\ \mathrm{H} & 1.38017 & 2.77015 & 2.27200 \\ \mathrm{H} & 0.90713 & 4.86384 & 1.01268 \\ \mathrm{H} & -0.35318 & 4.74671 & -1.13237 \\ \mathrm{H} & -1.13607 & 2.56717 & -1.99032 \\ \mathrm{C} & -3.39195 & -1.91641 & -0.75783 \\ \mathrm{H} & -4.39409 & -1.73496 & -0.35966\end{array}$




$\begin{array}{lccc}\mathrm{H} & -3.38268 & -1.63990 & -1.81803 \\ \mathrm{H} & -3.12905 & -2.97327 & -0.68903 \\ \mathrm{C} & -1.85336 & -1.47314 & 1.85316 \\ \mathrm{H} & -2.73211 & -1.26688 & 2.47083 \\ \mathrm{H} & -1.61834 & -2.53893 & 1.85477 \\ \mathrm{H} & -0.99957 & -0.93653 & 2.27896 \\ \mathrm{C} & -3.25942 & 0.54107 & 0.54205 \\ \mathrm{H} & -4.14129 & 0.19815 & 1.09624 \\ \mathrm{H} & -2.74045 & 1.28852 & 1.15139 \\ \mathrm{H} & -3.60683 & 1.04356 & -0.36952\end{array}$

Cis TS3

$\mathrm{E}(\mathrm{B} 3 \mathrm{LYP}$ 6-31G(d)) Energy= -1076.98142270 Hartrees Imaginary Freq $=-441.4401 \mathrm{~cm}^{-1}$

$\begin{array}{lccc}\mathrm{O} & 0.80430 & -1.87319 & 0.00659 \\ \mathrm{C} & -0.14670 & -0.91971 & 1.01872 \\ \mathrm{P} & 2.23392 & -1.18953 & -0.13570 \\ \mathrm{C} & 0.49314 & 0.36470 & 1.16805 \\ \mathrm{C} & -1.55035 & -1.09403 & 0.47757 \\ \mathrm{C} & 0.17347 & 1.59794 & 0.46592 \\ \mathrm{C} & -1.90336 & -2.04781 & -0.48524 \\ \mathrm{C} & -3.23634 & -2.20752 & -0.87179 \\ \mathrm{C} & -4.23796 & -1.42824 & -0.29344 \\ \mathrm{C} & -3.89618 & -0.48079 & 0.67383 \\ \mathrm{C} & -2.56522 & -0.31299 & 1.05204 \\ \mathrm{C} & -0.52774 & 1.67295 & -0.76499 \\ \mathrm{C} & -0.75024 & 2.89095 & -1.40522 \\ \mathrm{C} & -0.27896 & 4.08897 & -0.86347 \\ \mathrm{C} & 0.42095 & 4.04196 & 0.34612 \\ \mathrm{C} & 0.63793 & 2.82993 & 0.99360 \\ \mathrm{H} & -0.02698 & -1.52957 & 1.91766 \\ \mathrm{H} & 0.97153 & 0.51393 & 2.13486 \\ \mathrm{H} & -1.12947 & -2.65976 & -0.93284 \\ \mathrm{H} & -3.48981 & -2.94868 & -1.62593 \\ \mathrm{H} & -5.27477 & -1.55564 & -0.59392 \\ \mathrm{H} & -4.66591 & 0.13690 & 1.12940 \\ \mathrm{H} & -2.30026 & 0.43565 & 1.79265 \\ \mathrm{H} & -0.90960 & 0.76438 & -1.21997 \\ \mathrm{H} & -1.30043 & 2.90050 & -2.34413 \\ \mathrm{H} & -0.45396 & 5.03494 & -1.36850\end{array}$




$\begin{array}{lccc}\mathrm{H} & 0.79507 & 4.96039 & 0.79429 \\ \mathrm{H} & 1.17496 & 2.82063 & 1.94113 \\ \mathrm{C} & 3.05168 & -2.55399 & -1.06186 \\ \mathrm{H} & 4.09984 & -2.31303 & -1.27132 \\ \mathrm{H} & 3.00627 & -3.47923 & -0.48085 \\ \mathrm{H} & 2.53041 & -2.71970 & -2.00904 \\ \mathrm{C} & 2.50871 & 0.25057 & -1.22839 \\ \mathrm{H} & 3.34832 & 0.05173 & -1.90397 \\ \mathrm{H} & 1.60510 & 0.43377 & -1.81612 \\ \mathrm{H} & 2.69476 & 1.15103 & -0.64163 \\ \mathrm{C} & 3.28851 & -1.04187 & 1.35925 \\ \mathrm{H} & 4.31198 & -1.36023 & 1.12613 \\ \mathrm{H} & 3.29164 & -0.01759 & 1.73187 \\ \mathrm{H} & 2.88992 & -1.69789 & 2.13888\end{array}$

Cis stilbene I

E(B3LYP 6-31G(d)) Energy=-540.701966809 Hartrees Number of imaginary frequencies $=0$

$\begin{array}{lccc}\mathrm{C} & -0.674885 & 1.823991 & 0.000227 \\ \mathrm{C} & 0.674828 & 1.824087 & 0.000049 \\ \mathrm{C} & -1.646896 & 0.716617 & -0.066994 \\ \mathrm{C} & -1.416882 & -0.467650 & -0.791517 \\ \mathrm{C} & -2.390178 & -1.461550 & -0.862054 \\ \mathrm{C} & -3.617986 & -1.297786 & -0.214954 \\ \mathrm{C} & -3.869061 & -0.121581 & 0.493217 \\ \mathrm{C} & -2.898065 & 0.876464 & 0.555930 \\ \mathrm{H} & -1.141822 & 2.808411 & 0.041910 \\ \mathrm{H} & 1.141658 & 2.808580 & -0.041136 \\ \mathrm{H} & -0.473840 & -0.599953 & -1.311268 \\ \mathrm{H} & -2.192533 & -2.365339 & -1.432720 \\ \mathrm{H} & -4.375149 & -2.075183 & -0.272185 \\ \mathrm{H} & -4.824169 & 0.022830 & 0.991543 \\ \mathrm{H} & -3.103809 & 1.795020 & 1.101034 \\ \mathrm{C} & 3.617984 & -1.297641 & 0.214858 \\ \mathrm{C} & 3.868785 & -0.121732 & -0.493894 \\ \mathrm{C} & 2.897792 & 0.876332 & -0.556585 \\ \mathrm{C} & 1.646944 & 0.716738 & 0.066998 \\ \mathrm{C} & 1.417178 & -0.467224 & 0.792091 \\ \mathrm{C} & 2.390469 & -1.461139 & 0.862580 \\ \mathrm{H} & 4.375173 & -2.075013 & 0.272105\end{array}$




$\begin{array}{cccc}\mathrm{H} & 4.823664 & 0.022425 & -0.992731 \\ \mathrm{H} & 3.103295 & 1.794653 & -1.102172 \\ \mathrm{H} & 0.474324 & -0.599262 & 1.312259 \\ \mathrm{H} & 2.193057 & -2.364724 & 1.433649\end{array}$

Trans Adduct $\left(\mathrm{Me}_{3} \mathrm{PCHPh}+\mathrm{PhCHO}\right)$

E(B3LYP 6-31G(d)) Energy=-1076.99840985 Hartrees

Number of imaginary frequencies $=0$

$\begin{array}{lccc}\mathrm{O} & -1.24369 & -2.15745 & 1.45114 \\ \mathrm{C} & -1.39195 & -0.95249 & 1.28470 \\ \mathrm{P} & 1.90676 & -1.68004 & -0.38404 \\ \mathrm{C} & 1.09210 & -0.22496 & -0.73949 \\ \mathrm{C} & -2.54481 & -0.34660 & 0.58605 \\ \mathrm{C} & 1.47054 & 1.11576 & -0.35828 \\ \mathrm{C} & -2.60290 & 1.04439 & 0.41846 \\ \mathrm{C} & -3.69356 & 1.62343 & -0.22940 \\ \mathrm{C} & -4.72917 & 0.81553 & -0.70471 \\ \mathrm{C} & -4.67587 & -0.57376 & -0.53765 \\ \mathrm{C} & -3.58748 & -1.15421 & 0.10433 \\ \mathrm{C} & 2.57314 & 1.43509 & 0.47638 \\ \mathrm{C} & 2.87729 & 2.74851 & 0.82421 \\ \mathrm{C} & 2.10341 & 3.81467 & 0.36011 \\ \mathrm{C} & 1.01433 & 3.52970 & -0.46954 \\ \mathrm{C} & 0.70542 & 2.21983 & -0.82076 \\ \mathrm{H} & -0.65394 & -0.23100 & 1.68125 \\ \mathrm{H} & 0.26546 & -0.34593 & -1.43363 \\ \mathrm{H} & -1.78483 & 1.66310 & 0.77912 \\ \mathrm{H} & -3.73694 & 2.70065 & -0.36351 \\ \mathrm{H} & -5.58106 & 1.26719 & -1.20647 \\ \mathrm{H} & -5.48567 & -1.19632 & -0.90856 \\ \mathrm{H} & -3.52177 & -2.22827 & 0.24987 \\ \mathrm{H} & 3.21155 & 0.64105 & 0.85910 \\ \mathrm{H} & 3.73447 & 2.93987 & 1.46680 \\ \mathrm{H} & 2.34464 & 4.83806 & 0.63270 \\ \mathrm{H} & 0.39887 & 4.34069 & -0.85441 \\ \mathrm{H} & -0.14150 & 2.02673 & -1.47600 \\ \mathrm{C} & 0.94514 & -3.06511 & -1.08753 \\ \mathrm{H} & 1.49288 & -4.00474 & -0.96940 \\ \mathrm{H} & -0.00955 & -3.13519 & -0.55890 \\ \mathrm{H} & 0.76337 & -2.88903 & -2.15140 \\ & & & \\ & & & \\ & & & \end{array}$




$\begin{array}{llll}\mathrm{C} & 3.62421 & -1.87168 & -1.05278 \\ \mathrm{H} & 4.08638 & -2.81172 & -0.73096 \\ \mathrm{H} & 3.58429 & -1.83755 & -2.14555 \\ \mathrm{H} & 4.24117 & -1.03555 & -0.70996 \\ \mathrm{C} & 2.10776 & -2.10516 & 1.39911 \\ \mathrm{H} & 2.72317 & -3.00311 & 1.52180 \\ \mathrm{H} & 2.58017 & -1.27666 & 1.93373 \\ \mathrm{H} & 1.11133 & -2.27955 & 1.81656\end{array}$

Trans TS1

E(B3LYP 6-31G(d)) Energy= -1076.98381841 Hartrees Imaginary Freq $=-213.6518 \mathrm{~cm}^{-1}$

$\begin{array}{lccc}\mathrm{O} & -0.348517 & -2.338169 & 1.087326 \\ \mathrm{C} & -0.588090 & -1.079639 & 0.989744 \\ \mathrm{P} & 2.000013 & -1.366979 & -0.282815 \\ \mathrm{C} & 0.569031 & -0.287328 & -0.333889 \\ \mathrm{C} & -1.958454 & -0.644608 & 0.495596 \\ \mathrm{C} & 0.800167 & 1.171871 & -0.201013 \\ \mathrm{C} & -2.455282 & 0.645701 & 0.717829 \\ \mathrm{C} & -3.723520 & 1.007330 & 0.259024 \\ \mathrm{C} & -4.512229 & 0.079691 & -0.425179 \\ \mathrm{C} & -4.028117 & -1.214297 & -0.638029 \\ \mathrm{C} & -2.762173 & -1.572826 & -0.176808 \\ \mathrm{C} & 1.505999 & 1.729315 & 0.885517 \\ \mathrm{C} & 1.662716 & 3.109365 & 1.017616 \\ \mathrm{C} & 1.104150 & 3.977616 & 0.078671 \\ \mathrm{C} & 0.382610 & 3.445813 & -0.993431 \\ \mathrm{C} & 0.236260 & 2.067807 & -1.131976 \\ \mathrm{H} & -0.251225 & -0.414176 & 1.819188 \\ \mathrm{H} & 0.046312 & -0.532415 & -1.262615 \\ \mathrm{H} & -1.849533 & 1.367288 & 1.260862 \\ \mathrm{H} & -4.099011 & 2.011499 & 0.441969 \\ \mathrm{H} & -5.501706 & 0.359139 & -0.778981 \\ \mathrm{H} & -4.645057 & -1.946277 & -1.154820 \\ \mathrm{H} & -2.375103 & -2.579867 & -0.300559 \\ \mathrm{H} & 1.916391 & 1.078800 & 1.653611 \\ \mathrm{H} & 2.214173 & 3.506290 & 1.866489 \\ \mathrm{H} & 1.221963 & 5.052451 & 0.184510 \\ \mathrm{H} & -0.066723 & 4.108434 & -1.729028 \\ \mathrm{H} & & & -1.970034 \\ & & & \end{array}$




$\begin{array}{llll}\mathrm{C} & 1.560623 & -2.927753 & -1.107290 \\ \mathrm{H} & 2.371553 & -3.657571 & -1.020822 \\ \mathrm{H} & 0.661220 & -3.274704 & -0.585901 \\ \mathrm{H} & 1.344637 & -2.744357 & -2.164267 \\ \mathrm{C} & 3.452963 & -0.661800 & -1.153287 \\ \mathrm{H} & 4.313613 & -1.334459 & -1.074030 \\ \mathrm{H} & 3.212391 & -0.508298 & -2.209229 \\ \mathrm{H} & 3.708579 & 0.306082 & -0.713042 \\ \mathrm{C} & 2.568417 & -1.784606 & 1.401774 \\ \mathrm{H} & 3.378758 & -2.518457 & 1.341846 \\ \mathrm{H} & 2.932031 & -0.896638 & 1.924764 \\ \mathrm{H} & 1.694010 & -2.211476 & 1.904486\end{array}$

Trans OP1

E(B3LYP 6-31G(d)) Energy=-1077.01021317 Hartrees Number of imaginary frequencies $=0$

$\begin{array}{lccc}\mathrm{O} & -0.796569 & -1.694556 & 0.879900 \\ \mathrm{C} & -0.772149 & -0.289160 & 0.789934 \\ \mathrm{P} & 0.773408 & -1.993360 & -0.071613 \\ \mathrm{C} & 0.342750 & -0.142900 & -0.278906 \\ \mathrm{C} & -2.105024 & 0.328135 & 0.402149 \\ \mathrm{C} & 1.405691 & 0.913645 & -0.162075 \\ \mathrm{C} & -2.284824 & 1.716524 & 0.471918 \\ \mathrm{C} & -3.492921 & 2.297355 & 0.087411 \\ \mathrm{C} & -4.543531 & 1.494813 & -0.365071 \\ \mathrm{C} & -4.374750 & 0.110856 & -0.426789 \\ \mathrm{C} & -3.162558 & -0.468996 & -0.045857 \\ \mathrm{C} & 2.026764 & 1.209909 & 1.063800 \\ \mathrm{C} & 3.020992 & 2.184235 & 1.149729 \\ \mathrm{C} & 3.419512 & 2.886108 & 0.010441 \\ \mathrm{C} & 2.810557 & 2.607679 & -1.214017 \\ \mathrm{C} & 1.814430 & 1.634744 & -1.295170 \\ \mathrm{H} & -0.447025 & 0.174905 & 1.737521 \\ \mathrm{H} & -0.117662 & -0.077351 & -1.273925 \\ \mathrm{H} & -1.472411 & 2.346156 & 0.829848 \\ \mathrm{H} & -3.616914 & 3.375980 & 0.145655 \\ \mathrm{H} & -5.487702 & 1.945692 & -0.659859 \\ \mathrm{H} & -5.191389 & -0.521482 & -0.767229 \\ \mathrm{H} & -3.023584 & -1.544611 & -0.072354 \\ \mathrm{H} & 1.722966 & 0.682991 & 1.964373\end{array}$




$\begin{array}{lccc}\mathrm{H} & 3.483544 & 2.396633 & 2.110116 \\ \mathrm{H} & 4.193073 & 3.646216 & 0.078017 \\ \mathrm{H} & 3.106127 & 3.152099 & -2.107113 \\ \mathrm{H} & 1.340109 & 1.430950 & -2.252467 \\ \mathrm{C} & -0.088370 & -3.371678 & -0.953688 \\ \mathrm{H} & 0.620553 & -4.091827 & -1.371694 \\ \mathrm{H} & -0.782054 & -3.859627 & -0.266974 \\ \mathrm{H} & -0.674604 & -2.941971 & -1.773403 \\ \mathrm{C} & 2.300307 & -1.985520 & -1.209032 \\ \mathrm{H} & 2.762687 & -2.978236 & -1.262652 \\ \mathrm{H} & 2.020200 & -1.689052 & -2.227980 \\ \mathrm{H} & 3.052909 & -1.273541 & -0.854380 \\ \mathrm{C} & 1.649866 & -2.575020 & 1.442197 \\ \mathrm{H} & 2.453277 & -3.273242 & 1.189923 \\ \mathrm{H} & 2.097693 & -1.712162 & 1.946535 \\ \mathrm{H} & 0.931186 & -3.036057 & 2.121203\end{array}$

Trans TS2

E(B3LYP 6-31G(d)) Energy= -1077.00572671 Hartrees Imaginary Freq $=-37.3951 \mathrm{~cm}^{-1}$

$\begin{array}{lccc}\mathrm{O} & -0.61515 & 1.21184 & -1.14817 \\ \mathrm{C} & -0.62010 & -0.17807 & -0.78253 \\ \mathrm{P} & 0.30184 & 1.86759 & 0.19651 \\ \mathrm{C} & 0.41098 & -0.12802 & 0.35306 \\ \mathrm{C} & -2.00333 & -0.68274 & -0.40527 \\ \mathrm{C} & 1.73611 & -0.78598 & 0.10459 \\ \mathrm{C} & -2.15334 & -1.94513 & 0.18662 \\ \mathrm{C} & -3.41853 & -2.43431 & 0.50999 \\ \mathrm{C} & -4.55604 & -1.66753 & 0.24495 \\ \mathrm{C} & -4.41615 & -0.41397 & -0.35112 \\ \mathrm{C} & -3.14829 & 0.07360 & -0.67819 \\ \mathrm{C} & 2.38724 & -0.69751 & -1.13982 \\ \mathrm{C} & 3.62930 & -1.29556 & -1.34720 \\ \mathrm{C} & 4.25785 & -1.99497 & -0.31406 \\ \mathrm{C} & 3.62776 & -2.09131 & 0.92708 \\ \mathrm{C} & 2.38238 & -1.49521 & 1.12994 \\ \mathrm{H} & -0.25714 & -0.76488 & -1.63730 \\ \mathrm{H} & 0.01224 & -0.45789 & 1.31879 \\ \mathrm{H} & -1.27242 & -2.54865 & 0.39509 \\ \mathrm{H} & -3.51683 & -3.41384 & 0.97129\end{array}$




$\begin{array}{lccc}\mathrm{H} & -5.54239 & -2.04685 & 0.49898 \\ \mathrm{H} & -5.29604 & 0.18713 & -0.56716 \\ \mathrm{H} & -3.03209 & 1.04347 & -1.15092 \\ \mathrm{H} & 1.91613 & -0.15107 & -1.95398 \\ \mathrm{H} & 4.10910 & -1.21363 & -2.31932 \\ \mathrm{H} & 5.22588 & -2.46107 & -0.47664 \\ \mathrm{H} & 4.10161 & -2.63788 & 1.73867 \\ \mathrm{H} & 1.89599 & -1.58566 & 2.09921 \\ \mathrm{C} & -0.82055 & 2.24206 & 1.61284 \\ \mathrm{H} & -0.78124 & 3.30777 & 1.85813 \\ \mathrm{H} & -1.84673 & 1.97880 & 1.34119 \\ \mathrm{H} & -0.53256 & 1.66043 & 2.49303 \\ \mathrm{C} & 2.00928 & 2.19303 & 0.91823 \\ \mathrm{H} & 2.09928 & 3.23174 & 1.25205 \\ \mathrm{H} & 2.20400 & 1.51500 & 1.75441 \\ \mathrm{H} & 2.77726 & 2.00779 & 0.15957 \\ \mathrm{C} & 0.25712 & 3.49783 & -0.75031 \\ \mathrm{H} & 0.56563 & 4.32925 & -0.10841 \\ \mathrm{H} & 0.94122 & 3.44886 & -1.60442 \\ \mathrm{H} & -0.74744 & 3.68531 & -1.13737\end{array}$

Trans OP2

E(B3LYP 6-31G(d)) Energy=-1077.00616782 Hartrees Number of imaginary frequencies $=0$

$\begin{array}{lccc}\mathrm{O} & -0.722807 & 1.314962 & -1.060119 \\ \mathrm{C} & -0.647206 & -0.103668 & -0.777735 \\ \mathrm{P} & 0.298987 & 1.959191 & 0.158148 \\ \mathrm{C} & 0.404069 & -0.080468 & 0.331612 \\ \mathrm{C} & -2.001125 & -0.679008 & -0.401738 \\ \mathrm{C} & 1.698493 & -0.796110 & 0.107849 \\ \mathrm{C} & -3.180132 & 0.055565 & -0.566781 \\ \mathrm{C} & -4.416239 & -0.502246 & -0.229755 \\ \mathrm{C} & -4.489960 & -1.802784 & 0.268708 \\ \mathrm{C} & -3.317483 & -2.546356 & 0.427307 \\ \mathrm{C} & -2.084054 & -1.988156 & 0.094793 \\ \mathrm{C} & 2.361008 & -1.405151 & 1.189282 \\ \mathrm{C} & 3.590404 & -2.043181 & 1.025819 \\ \mathrm{C} & 4.192231 & -2.098463 & -0.232337 \\ \mathrm{C} & 3.548847 & -1.504905 & -1.320481 \\ \mathrm{C} & 2.323640 & -0.861132 & -1.152153\end{array}$




$\begin{array}{lccc}\mathrm{H} & -0.288483 & -0.610157 & -1.682538 \\ \mathrm{H} & -0.014619 & -0.386374 & 1.295859 \\ \mathrm{H} & -3.119669 & 1.064040 & -0.962243 \\ \mathrm{H} & -5.323382 & 0.082585 & -0.360988 \\ \mathrm{H} & -5.451751 & -2.236080 & 0.530418 \\ \mathrm{H} & -3.363602 & -3.561501 & 0.813590 \\ \mathrm{H} & -1.174794 & -2.571282 & 0.223926 \\ \mathrm{H} & 1.896615 & -1.382074 & 2.173309 \\ \mathrm{H} & 4.073684 & -2.506842 & 1.882369 \\ \mathrm{H} & 5.146922 & -2.600191 & -0.364548 \\ \mathrm{H} & 4.003639 & -1.541321 & -2.307406 \\ \mathrm{H} & 1.848392 & -0.399839 & -2.014721 \\ \mathrm{C} & -0.320988 & 2.231689 & 1.884830 \\ \mathrm{H} & -0.286578 & 3.289448 & 2.162010 \\ \mathrm{H} & -1.361367 & 1.891911 & 1.934284 \\ \mathrm{H} & 0.265006 & 1.641458 & 2.594851 \\ \mathrm{C} & 2.130646 & 2.278967 & 0.167869 \\ \mathrm{H} & 2.629903 & 1.635391 & 0.895539 \\ \mathrm{H} & 2.546199 & 2.046868 & -0.818205 \\ \mathrm{H} & 2.331420 & 3.331124 & 0.388491 \\ \mathrm{C} & -0.169155 & 3.660782 & -0.527032 \\ \mathrm{H} & 0.286905 & 4.459775 & 0.067857 \\ \mathrm{H} & 0.170807 & 3.761084 & -1.563229 \\ \mathrm{H} & -1.255575 & 3.792164 & -0.520245\end{array}$

Trans TS3

E(B3LYP 6-31G(d)) Energy= -1076.98730242 Hartrees Imaginary Freq $=-433.9648 \mathrm{~cm}^{-1}$

$\begin{array}{lccc}\mathrm{O} & -0.681182 & 1.253440 & -0.983911 \\ \mathrm{C} & -0.527924 & -0.383338 & -0.630155 \\ \mathrm{P} & 0.028338 & 2.078154 & 0.180552 \\ \mathrm{C} & 0.478892 & -0.523559 & 0.388324 \\ \mathrm{C} & -1.942934 & -0.830189 & -0.334238 \\ \mathrm{C} & 1.852632 & -0.901638 & 0.101179 \\ \mathrm{C} & -3.050914 & -0.230742 & -0.955582 \\ \mathrm{C} & -4.342305 & -0.702696 & -0.724045 \\ \mathrm{C} & -4.554037 & -1.789833 & 0.127007 \\ \mathrm{C} & -3.461253 & -2.402326 & 0.739465 \\ \mathrm{C} & -2.167945 & -1.928247 & 0.508923 \\ \mathrm{C} & 2.696124 & -1.355179 & 1.146531\end{array}$




$\begin{array}{lccc}\mathrm{C} & 4.024901 & -1.701589 & 0.924446 \\ \mathrm{C} & 4.582134 & -1.616884 & -0.355636 \\ \mathrm{C} & 3.773776 & -1.171949 & -1.404153 \\ \mathrm{C} & 2.444696 & -0.814250 & -1.184334 \\ \mathrm{H} & -0.223472 & -0.684189 & -1.635400 \\ \mathrm{H} & 0.125451 & -0.781467 & 1.384627 \\ \mathrm{H} & -2.891446 & 0.617374 & -1.612443 \\ \mathrm{H} & -5.186059 & -0.220344 & -1.211385 \\ \mathrm{H} & -5.560960 & -2.157818 & 0.306222 \\ \mathrm{H} & -3.610744 & -3.254608 & 1.397480 \\ \mathrm{H} & -1.320705 & -2.422789 & 0.972679 \\ \mathrm{H} & 2.280720 & -1.446338 & 2.149180 \\ \mathrm{H} & 4.631040 & -2.050111 & 1.758335 \\ \mathrm{H} & 5.618865 & -1.890601 & -0.530325 \\ \mathrm{H} & 4.182861 & -1.096965 & -2.409828 \\ \mathrm{H} & 1.852794 & -0.461051 & -2.026084 \\ \mathrm{C} & -0.658611 & 1.951637 & 1.877276 \\ \mathrm{H} & -0.842894 & 2.951815 & 2.287484 \\ \mathrm{H} & -1.606669 & 1.409159 & 1.824801 \\ \mathrm{H} & 0.021817 & 1.399652 & 2.526242 \\ \mathrm{C} & 1.847551 & 2.270058 & 0.240387 \\ \mathrm{H} & 2.263942 & 1.729513 & 1.091666 \\ \mathrm{H} & 2.283768 & 1.831028 & -0.661360 \\ \mathrm{H} & 2.112332 & 3.332023 & 0.294093 \\ \mathrm{C} & -0.525245 & 3.751016 & -0.354087 \\ \mathrm{H} & -0.149365 & 4.523564 & 0.326135 \\ \mathrm{H} & -0.156358 & 3.957497 & -1.362938 \\ \mathrm{H} & -1.617762 & 3.793072 & -0.374007\end{array}$

Trans stilbene

E(B3LYP 6-31G(d)) Energy=-540.709964725 Hartrees Number of imaginary frequencies $=0$

$\begin{array}{lccc}\mathrm{C} & -0.498623 & 0.454057 & 0.001393 \\ \mathrm{C} & 0.498604 & -0.453938 & 0.000401 \\ \mathrm{C} & -1.940013 & 0.186538 & 0.002143 \\ \mathrm{C} & -2.829299 & 1.276762 & -0.043083 \\ \mathrm{C} & -4.209795 & 1.087548 & -0.046622 \\ \mathrm{C} & -4.739032 & -0.202647 & -0.003663 \\ \mathrm{C} & -3.871544 & -1.298557 & 0.043705 \\ \mathrm{C} & -2.493570 & -1.108604 & 0.047326\end{array}$




$\begin{array}{lccc}\mathrm{H} & -0.242556 & 1.512671 & -0.003635 \\ \mathrm{H} & 0.242496 & -1.512532 & -0.006681 \\ \mathrm{H} & -2.424556 & 2.285856 & -0.077034 \\ \mathrm{H} & -4.871836 & 1.948788 & -0.082658 \\ \mathrm{H} & -5.814783 & -0.355373 & -0.005650 \\ \mathrm{H} & -4.273380 & -2.307896 & 0.079645 \\ \mathrm{H} & -1.840869 & -1.975600 & 0.088924 \\ \mathrm{C} & 4.739047 & 0.202547 & -0.003224 \\ \mathrm{C} & 4.209761 & -1.087618 & -0.046287 \\ \mathrm{C} & 2.829248 & -1.276753 & -0.043300 \\ \mathrm{C} & 1.940005 & -0.186484 & 0.001557 \\ \mathrm{C} & 2.493617 & 1.108642 & 0.046707 \\ \mathrm{C} & 3.871596 & 1.298516 & 0.043620 \\ \mathrm{H} & 5.814805 & 0.355228 & -0.004824 \\ \mathrm{H} & 4.871768 & -1.948898 & -0.081982 \\ \mathrm{H} & 2.424463 & -2.285830 & -0.077313 \\ \mathrm{H} & 1.840949 & 1.975690 & 0.087672 \\ \mathrm{H} & 4.273483 & 2.307838 & 0.079488\end{array}$

$\mathrm{Me}_{3} \mathrm{PO}$

E(B3LYP 6-31G(d)) Energy=-536.352997260 Hartrees Number of imaginary frequencies $=0$

$\begin{array}{lccc}\mathrm{O} & 0.001008 & 0.000418 & 1.693201 \\ \mathrm{P} & 0.000071 & 0.000086 & 0.191899 \\ \mathrm{C} & 1.672851 & -0.004208 & -0.562389 \\ \mathrm{C} & -0.840426 & -1.447038 & -0.560914 \\ \mathrm{C} & -0.833397 & 1.450803 & -0.561508 \\ \mathrm{H} & 2.222556 & 0.879956 & -0.224835 \\ \mathrm{H} & 1.631420 & -0.003535 & -1.657039 \\ \mathrm{H} & 2.216965 & -0.892259 & -0.225888 \\ \mathrm{H} & -0.821824 & -1.410760 & -1.655610 \\ \mathrm{H} & -1.880705 & -1.475732 & -0.221981 \\ \mathrm{H} & -0.347655 & -2.364780 & -0.225058 \\ \mathrm{H} & -1.873739 & 1.484519 & -0.223266 \\ \mathrm{H} & -0.813943 & 1.414448 & -1.656164 \\ \mathrm{H} & -0.336368 & 2.366158 & -0.225386\end{array}$

